\title{
Deep spatial profiling of COVID19 brains reveals neuroinflammation by compartmentalized local immune cell interactions and targets for intervention
}

Bertram Bengsch ( $\nabla$ bertram.bengsch@uniklinik-freiburg.de)

University Medical Center Freiburg https://orcid.org/0000-0003-2552-740X

Marius Schwabenland

Institute of Neuropathology, Faculty of Medicine, University of Freiburg https://orcid.org/0000-0003-

2205-5427

Henrike Salié

University Medical Center Freiburg

Jovan Tanevski

Heidelberg University Hospital https://orcid.org/0000-0001-7177-1003

Saskia Killmer

Department of Medicine II, Freiburg Freiburg University Medical Center, Faculty of Medicine, University of

Freiburg, Freiburg, Germany

Jakob Matschke

University Medical Center Hamburg-Eppendorf

Klaus Püschel

University Medical Center Hamburg-Eppendorf

Henrik Mei

German Rheumatism Research Center Berlin (DRFZ), a Leibniz Institute, Berlin, Germany

\section{Tobias Boettler}

Department of Medicine II (Gastroenterology, Hepatology, Endocrinology and Infectious Diseases),

Freiburg University Medical Center, Faculty of Medicine, University of Freiburg, Freiburg, Germany

https://orcid.org/0000-0002-1195-055X

\section{Christoph Neumann-Haefelin}

Department of Medicine II (Gastroenterology, Hepatology, Endocrinology and Infectious Diseases),

Freiburg University Medical Center, Faculty of Medicine, University of Freiburg, Freiburg, Germany

\section{Maike Hofmann}

University Hospital Freiburg https://orcid.org/0000-0001-8410-8833

Julio Saez-Rodriguez

Heidelberg University Hospital

Klaus-Peter Knobeloch 
University of Freiburg https://orcid.org/0000-0003-1587-1944

\section{Thomas Blank}

University of Freiburg

\section{Robert Thimme}

Department of Medicine II, Freiburg Freiburg University Medical Center, Faculty of Medicine, University of Freiburg, Freiburg, Germany https://orcid.org/0000-0003-1417-4135

\section{Markus Glatzel}

University Medical Center Hamburg-Eppendorf https://orcid.org/0000-0002-7720-8817

\section{Marco Prinz ( $\square$ marco.prinz@uniklinik-freiburg.de)}

University of Freiburg https://orcid.org/0000-0002-0349-1955

\section{Research Article}

Keywords: SARS-CoV-2, COVID-19, encephalopathy, brain autopsy, microglia, T cells, high-dimensional imaging, neuroinflammation, neurological deficits, machine learning, single-cell analysis, mass cytometry

Posted Date: August 28th, 2020

DOI: https://doi.org/10.21203/rs.3.rs-63687/v1

License: (c) (i) This work is licensed under a Creative Commons Attribution 4.0 International License. Read Full License 


\section{Deep spatial profiling of COVID19 brains reveals neuroinflammation by compartmentalized local immune cell interactions and targets for intervention}

Marius Schwabenland ${ }^{1 *}$, Henrike Salié2*, Jovan Tanevski ${ }^{3}$, Saskia Killmer ${ }^{2}$, Jakob Matschke ${ }^{4}$, Klaus Püschel ${ }^{5}$, Henrik E. Mei ${ }^{6}$, Tobias Boettler ${ }^{2}$, Christoph Neumann-Haefelin², Maike Hofmann², Julio Saez-Rodriguez ${ }^{3}$, Klaus-Peter Knobeloch ${ }^{1}$, Thomas Blank ${ }^{1}$, Robert Thimme ${ }^{2}$, Markus Glatzel $^{4}$, Marco Prinz ${ }^{1,7,8 \#} \&$ Bertram Bengsch ${ }^{2,8 \#}$

* equal contribution first authors \# equal contribution last authors

${ }^{1}$ Institute of Neuropathology, Faculty of Medicine, University of Freiburg, Freiburg, Germany ${ }^{2}$ Faculty of Medicine, Department of Medicine II, Gastroenterology, Hepatology, Endocrinology, and Infectious Disease, University Medical Center Freiburg, Freiburg, Germany

${ }^{3}$ Institute for Computational Biomedicine, Faculty of Medicine, Heidelberg University and Heidelberg University Hospital, Heidelberg, Germany

${ }^{4}$ Institute of Neuropathology, University Medical Center Hamburg-Eppendorf, Hamburg,

Germany

${ }^{5}$ Institute of Legal Medicine, University Medical Center Hamburg-Eppendorf, Hamburg,

Germany

${ }^{6}$ German Rheumatism Research Center Berlin (DRFZ), a Leibniz Institute, Berlin, Germany ${ }^{7}$ Center for Basics in NeuroModulation (NeuroModulBasics), Faculty of Medicine, University of Freiburg, Freiburg, Germany

${ }^{8}$ Signaling Research Centers BIOSS and CIBSS, University of Freiburg, Freiburg, Germany

Correspondence to:

Marco Prinz

Institute of Neuropathology

University of Freiburg

Breisacher Str. 64

D-79106 Freiburg, Germany

Phone: +49-761-270-51050

E-mail:marco.prinz@uniklinik-freiburg.de or Bertram Bengsch

University Medical Center Freiburg

Department of Medicine II

Hugstetter Str. 55

D-79106 Freiburg, Germany

Phone: +49-761-270-32870

E-mail: Bertram.Bengsch@uniklinik-freiburg.de 


\section{ABSTRACT}

1 COVID-19 causes neurological symptoms that can be potentially life-threatening in up to $67 \%$ of

2 the patients. The underlying pathophysiological mechanisms of COVID-19 associated

3 encephalopathy, the involved immune cells, their spatial distribution and their cellular interactions

4 during disease remain largely unclear. In this study, we performed a 38-biomarker imaging mass

5 cytometry analysis of the brain stem from 25 patients and additional controls to understand the

6 local immune response during SARS-CoV-2 infection at a spatially resolved, high-dimensional

7 single-cell level. Importantly, utilizing an unbiased image segmentation and cell classification

8 pipeline, we observed a significant immune activation in the central nervous system (CNS) and

9 identified novel context-specific CD8 T cell and microglial clusters. Spatially resolved single-cell

10 analysis identified distinct phenotypes of $T$ cells and microglial clusters, their presence in specific

11 anatomical regions and their cellular interactions. Our analysis further highlights microglial

12 nodules and perivascular immune cell clusters as key sites of the local immune response. It also

13 demonstrates that disease-associated neuroinflammation is associated with severe axonal

14 damage as a structural basis for the neurologic deficits. Finally, we identified compartment- and

15 cluster-specific immune checkpoints that can be used for future therapeutic interventions.

\section{KEYWORDS}

22 SARS-CoV-2, COVID-19, encephalopathy, brain autopsy, microglia, T cells, high-dimensional 23 imaging, neuroinflammation, neurological deficits, machine learning, single-cell analysis, mass 24 cytometry 


\section{INTRODUCTION}

COVID-19 due to the SARS-CoV-2 pandemic is emerging as a multifaceted disease with multiorgan complications. One major complication are neurological symptoms that can occur during acute infection in up to $67 \%$ of the patients but also persist or even emerge after viral elimination and are summarized as COVID-19 encephalopathy ${ }^{1-3}$. Typically, COVID-19 encephalopathy manifests with anosmia but symptoms range from gait disorders, breathing difficulties to coma suggesting involvement of several brain regions such as the olfactory bulb but also infratentorial regions like the medulla ${ }^{4}$. Thromboembolic events including stroke were reported to be a major complication of COVID-19, but are unlikely to explain COVID-19 neurological symptoms in the majority of cases. Of note, SARS-CoV-2 has been detected in brain samples ${ }^{5}$ and immune activation in COVID-19 brains is debated based on differing observations from a limited number of small case series ${ }^{6-10}$, raising the possibility that immune-mediated mechanisms may drive COVID-19 encephalopathy. However, comprehensive data with detailed dissection of immune cell populations on a large collection of brains from COVID-19 patients is currently not yet available. Previous studies on peripheral immune functions in COVID-19 patients have uncovered patterns of immune activation and deviation in the peripheral blood and identified distinct immunotypes with differences in CD4 and CD8 activation, effector memory CD8 T cell differentiation and differential induction of $B$ cell responses that are linked to different disease severity ${ }^{11,12}$. In particular, an immunotype with strong CD4 T cell activation, and expansion of plasmablasts was linked to highest NIH COVID severity scales ${ }^{11,12}$. However, it is currently unclear whether the immune responses assessed in the peripheral blood reflect the immune responses in the tissue and which immune populations are involved in mediating organ pathology. Thus, in order to understand the cellular, immunological and anatomical basis of COVID-19 encephalopathy, we performed a highly multiplexed spatial analysis of the adaptive and innate immune system in brain sections from 25 COVID-19 patients that succumbed to the disease. We 
50 utilized imaging mass cytometry (IMC) that allowed the simultaneous detection of several key 51 immune populations, including novel disease-linked clusters of CD4 and CD8 T cells, NK cells, B 52 cells, and numerous myeloid and brain-specific innate immune populations, their cellular 53 interactions and importantly, their compartmentalization in distinct anatomical regions of the brain 54 stem. In addition, we performed a thorough molecular profiling of immune checkpoint receptor 55 expression together with immune activation and functional analyses. Spatial mapping of the 56 disease-associated altered brain immune response using imaging mass cytometry revealed 57 significant infiltration of distinct immune cell clusters with a prominent role for an activated CD8 T 58 cell cluster with concomitant expansion of microgliosis-related cell clusters in distinct anatomic 59 niches in the majority of patients. Our results indicate that during COVID-19 encephalopathy, the 60 adaptive as well as the CNS endogenous innate immune system are concomitantly activated, and 61 highlight disease-specific immune cell clusters that physically interact in distinct CNS 62 compartments. Compartment-enriched immune cell clusters revealed distinct immune marker 63 profiles that could be used for therapeutic interventions. Furthermore, the presence of immune 64 cell clusters correlates with the extent of axonal degeneration as a morphological correlate of 65 neuronal damage. Our data provide a foundation for novel therapeutic avenues aimed at reducing 66 neuroinflammation in COVID-19 patients. 
Highly multiplexed imaging of the brain microarchitecture by imaging mass cytometry indicates strong immune activation in COVID-19 patients

To understand the immune response in COVID-19-associated neuropathology, we performed

71 highly multiplexed high-dimensional imaging using imaging mass cytometry (IMC) together with

72 immunohistochemistry to identify the spatial brain immune profile in brain stem sections from

73 patients that succumbed to COVID-19 and compared it to control patients that died of non-

74 infectious and non-neurological reasons (Figure 1A). Classical immunohistochemistry for the

75 cytotoxic T-cell marker CD8 and the myeloid cell marker lba1 revealed significant CD8 T cell

76 infiltration and an elevated number of lba1 parenchymal cells with typical microglial morphology

77 (such as round to spindle-shaped somata and a distinct arborization pattern) indicating a robust

78 microgliosis (Figure 1B). Microgliosis occurred either in a diffuse manner or in clusters that are

79 referred to as microglia nodules. The observed CD8 immunoreactivity in 17 out of 25 patients and

80 pronounced microglia activation in 20 out of 25 patients suggested that classical

81 neuroinflammation ${ }^{13}$ is a histopathological hallmark of COVID-19-related neuropathology.

82 However, since classical immunohistochemistry allows analysis of only a few markers, limiting 83 insight into the fine composition of cellular phenotypes and immune cell subsets, we designed

84 and validated a 38-marker IMC approach to comprehensively profile key brain immune and 85 parenchymal cell populations. Tissue sections were immunolabeled with metal isotope coupled 86 antibodies. Spatial expression data was obtained after spot-by-spot laser ablation of the tissue at $871 \mu \mathrm{m}$ resolution and the subsequent mass spectrometry analysis of metal labels. Resolution of 88 IMC data was comparable to conventional immunohistochemistry (Figure 1B) but the high89 parametric information provided by this technique allowed deeper insights into the cellular 90 composition in specific anatomical compartments, such as areas with dense microglial 91 accumulation known as microglial nodules, that are absent in healthy brain tissue $e^{13,14}$. In a next 
92 step, expression of markers for canonical lymphoid and myeloid immune populations and 93 parenchymal brain cells was assessed together with markers of immune activation, function and 94 regulation (Figure 1C). Parallel manual count analysis indicated a significant immune infiltration 95 of the brain stem from COVID-19 patients by CD8 T cells and (albeit to a lower degree) CD4 T 96 cells and some B cells (Figure 1D). Importantly, this adaptive immune infiltration by immune cells 97 that are largely absent in control brains occurred in the presence of significantly increased 98 numbers of $\mathrm{Iba1}^{+}$microglial cells as well as $\mathrm{CD}_{163^{+}}$perivascular macrophages (Figure 1D). 99 Interestingly, we also observed a reduction in $\mathrm{CD}^{-} \mathrm{GzmB}^{+} \mathrm{NK}$ cells in diseased patients, indicative 100 of a $\sim 50 \%$ depletion of brain NK cells that has been described in the periphery of COVID-19 101 patients and is implicated in a more severe clinical course of COVID-19 ${ }^{15,16}$. Given the profound 102 immune activation in COVID-19 brains, we wondered whether this robust immune activation 103 pattern in the brain stem was linked to tissue destruction and neuronal damage. We thus 104 quantified amyloid precursor protein (APP) deposits in the brains of COVID-19 patients and 105 controls, respectively. Notably, significantly higher numbers of APP-positive deposits were observed in COVID-19 patients (Figure 1E). This damage occurred in the absence of an apparent 107 tissue necrosis, suggesting a specific and likely directed immune-mediated pathology.

108 Together, these analyses reveal strong signs of neuroinflammation with significant immune 109 infiltration, activation of the central nervous system (CNS) endogenous innate immune system 110 and accompanying increased neuronal damage in the brains of patients with COVID-19.

113 To understand the cellular phenotypes in the CNS during SARS-CoV-2 infection in greater detail, 114 we first utilized supervised machine learning to generate cell masks that allowed image 115 segmentation using a reference pipeline adapted for better resolution of microglial cells ${ }^{17}$ (Figure 
2A). We then extracted high-dimensional single-cell level data and clustered using Phenograph algorithm to identify the cellular composition in different patients (Figure 2A). Phenograph identifies cellular clusters on a graph constructed based on the high-dimensional phenotypic similarities of cells in the dataset before detecting discrete communities on this graph. It was chosen based on its robustness to identify even rare but phenotypically distinct populations ${ }^{18}$. This clustering strategy identified 31 distinct cellular clusters in the IMC analysis based on the high-dimensional segmented single cell data (Figure 2A). Most abundant clusters displayed molecular expression signatures of stromal (clusters c1, c4, c6, c8, c14, c21, c28), neuronal (c2, c20) and astrocytic (c3, c5, c18) cells, in agreement with the dominance of these cell types in the CNS (Figure 2B, Supplementary Figure 1A). Other identified clusters included cells with typical expression of endothelial cell markers (e.g. CD34, c9), or pericytes (c19). Importantly, this analysis also clearly identified multiple distinct immune clusters, such as several microglial clusters with varying expression of lba1 and markers of immune activation and lysosomal function such as HLA-DR and CD68 in clusters $c 7, c 11, c 12, c 13, c 15, c 16$, as well as different subsets of myeloid immune cells i.e. CD163+CD204+ perivascular macrophages (c27) and a related microglial cluster (c11). Lymphoid clusters detected included B cells, CD8 and CD4 T and NK cell subsets (clusters c23, c22, c29, c26, respectively) (Figure 2B). To understand changes in the immune landscape in COVID-19-linked neuroinflammation, we next visualized the immune cell clusters on a brain immune map generated via $t$-SNE and analyzed their abundance in patients and controls. This approach revealed major changes in the brain immune cell composition in the brains of COVID-19-affected patients, that were qualitatively and quantitatively dominated by activated microglial clusters (e.g., c7, c11, c13, c15, c16) and adaptive immune cell infiltration (c22, c23, c29) (Figures 2C-E). In contrast, only few immune clusters were present in control brains (e.g. c12, c26) and reduced in COVID-19 patients, suggesting a role in immune homeostasis for these microglial and NK cell populations. Taken together, our analyses identified 
141 distinct adaptive and innate immune cell clusters in the brain during SARS-CoV-2 infection that 142 were not present in control individuals.

144 The cellular interactome points to immune cell-crosstalk at the perivascular compartment during 145 COVID-19 neuroinflammation

146 We next wondered which cellular clusters were differentially enriched or depleted in the CNS of

147 COVID-19 patients across the cohort. As expected based on the analysis above, comparison of 148 high-dimensional clusters indicated a significant enrichment of CD8 T cell cluster c22, the CD4 T 149 cluster c29, and perivascular macrophage cluster c27 across COVID-19 patients (Figure 3A). 150 Interestingly, these data also revealed that COVID-19-associated neuroinflammation is linked to 151 significant expansion of an astrocytic (c3) and pericyte cluster (c19) (Figure 3A). Moreover, 152 SARS-CoV-2 receptor ACE2 expression was found on some brain vessels, suggesting the 153 possibility of viral antigens presented in the brain vasculature (Supplementary Figure 1B). 154 Therefore, it is tempting to speculate that COVID-19 immune pathology might be linked to uniform 155 vascular activation.

156 To understand co-regulated cell dynamics during COVID-19 encephalopathy in an unbiased 157 manner, we next performed a linear regression analysis between all clusters to determine highly 158 correlated immune infiltration patterns. This analysis identified modules of highly inter-correlated 159 immune cell clusters, including one that was composed of the clusters c3, c7, c22, c26, c27 and 160 c29, thus involving the major COVID-19-associated cellular phenotypes identified above (Figure 161 3B). As such, these patterns pointed to a close link between the vasculature-linked c27 and T cell 162 clusters c22 and c29, indicating T cell infiltration. We therefore asked if CD8 T cells were also 163 spatially linked to the perivascular compartment. Indeed, more than half of CD8 T cells in cluster 164 c22 were found in significantly closer proximity to vascular collagen-I in COVID-19 samples 
compared to a random distribution (Figure 3C). Neighborhood analysis further confirmed a close spatial interaction between c22 and vascular cell clusters, including endothelial cell cluster c9, pericyte cluster c19 and perivascular macrophage cluster c27 (Figure 3D). Importantly, the inflammatory spatial interaction pattern was pronounced in COVID-19 patients but not in controls, in which cluster c22 only displayed significant interactions with cells from the same cluster or homeostatic NK cell cluster c26 (Figure 3E, Supplementary Figure 1C). In sum, these data demonstrate a clear link between CD8 T cell infiltration and vascular activation during COVID-19related encephalitis.

\section{Activation of CD8 T cell cluster c22 in COVID-19 brains}

The strong enrichment of c22 representing CD8 T cells observed in COVID-19 brains pointed to a central role of this immune cell population. To understand their activation and function, we tested this cluster for the expression of markers informative about $\mathrm{T}$ cell differentiation, transcriptional programming, activation/exhaustion and function. We observed a strong enrichment of cluster c22 cells expressing CD45RO indicative of non-naive T cells (Figure 3F). Cells in c22 also expressed immune checkpoints PD-1 and Tim-3 as well as transcription factors Tox and Eomes, a phenotype compatible with highly activated effector cells with a possible induction of features of $\mathrm{T}$ cell exhaustion ${ }^{19}$. Of note, these cells also expressed granzyme $\mathrm{B}$, indicating cytotoxic effector T cell function (Figure 3F). In sum, the strong enrichment of CD8 T cells with significant activation involving several immune checkpoints in this immune cluster implicates that this immune cell population is a central mediator of neuroinflammation during SARS-CoV-2 infection.

\section{Immune activation in brains of COVID-19 patients occurs in distinct anatomic niches}


Our previous analysis indicated a strong perivascular immune infiltration linked to CD8 T cluster c22. However, importantly, we also noted that not all cells in this cluster were associated with the perivascular space (Figure 3C). Indeed, we also observed additional COVID-19-specific neighborhood relationships of CD8 T cluster c22 with microglial clusters c7, c11 and c15 outside 192 the perivascular region, in particular with microglial cluster c7 (Figure 3E), suggesting additional anatomic niches linked to specific microglial immune clusters in the brain parenchyma.

194 To systematically dissect these anatomic regions, we generated automated maps based on collagen-I and Iba1 expression that informed about the cellular distance to the vasculature and identified microglial hotspots (Figures 4A,B). Importantly, these analyses highlighted microglial nodules with an accumulation of densely packed microglial cells as an additional major site of infiltration by CD8 T cell cluster c22 (Figure 4C). Presence of microglial nodules was observed in the medulla of $28 \%$ of COVID-19 patients (7 out of 25 ) and in none of the control cases. Microglia nodules differed from more scattered parenchymal microgliosis observed in the majority of COVID-19 patients (76\%, 19 out of 25) and in $20 \%$ in the control cases (Figure 4C). These data highlighted the perivascular and microglial nodule compartment as key sites of COVID-19 immune responses. As shown exemplarily in Figure 4D, however, some additional CD8 T cell cluster c22 cells could also be identified scattered in the parenchyma. Thus, to dissect the role of distinct anatomic compartments as places of local immune responses, we performed a detailed cluster composition analysis in the perivascular, juxtavascular, parenchymal and microglial nodule compartments. Clearly, we identified major differences in the cluster composition of these compartments (Figure 4D). Most changes in cluster composition were found in microglial nodules 209 that were enriched in microglial clusters c7, c15 and CD8 T cell cluster c22 (Figure 4D,E). 210 Interestingly, in microglial nodules, only microglial clusters c7 and c15, but not microglial clusters 211 c11, c13, c16 were enriched, indicating context-specific roles for these microglial populations 212 (Figures 4D,E). In contrast, the perivascular compartment displayed enrichment of endothelial 
213 cell cluster c9, pericyte cluster c19 and perivascular macrophage cluster c27, with some 214 enrichment of CD8 T cell cluster c22, NK cell cluster c26 and stromal cluster c28 (Figures 4D,E).

215 Only subtle differences in cluster composition were found between the juxtavascular and 216 perivascular compartment, but their composition clearly differed from the nodule and perivascular 217 compartment (Figure 4D). These data illustrate differential immune activation consisting of 218 defined cell clusters at distinct anatomical regions in COVID-19-associated brains and identified 219 the key adaptive and innate immune subsets in each compartment.

222 As shown above, activated CD8 T cells in cluster c22 were significantly enriched in the CNS of 223 COVID-19 patients. However, the presence of heterogeneous immune cell subsets and their 224 different activation states at distinct anatomical niches suggested varying immunological roles for 225 these compartments. We next tested whether these anatomic compartments had an impact on 226 CD8 T cell activation and differentiation represented by changes in cluster c22. Interestingly, the 227 different anatomical regions were indeed linked to differences in T cell activation observed in c22.

228 The diffuse parenchymal compartment displayed the lowest levels of PD-1, CD39, Tim-3 and 229 Eomes in c22, while high expression of PD-1, CD39 and particularly Tim-3 was observed in cluster 230 c22 cells in the perivascular compartment (Figure 5A). These expression patterns differed from 231 c22 cells in microglial nodules, that displayed high co-expression of PD-1 and CD39, but not Tim2323 , however, the c22 cells in microglial nodules also featured higher induction of Eomes and HLA233 DR, indicating a more profound T-cell activation and a possible bias towards exhaustion. Of note, 234 while quantitative differences between the juxtavascular compartment and diffuse parenchyma 235 had not been observed based on cluster composition analysis (Figure 4D), qualitative differences 236 were apparent for CD8 T cell cluster c22, with elevated PD-1 expression in the juxtavascular 237 space (Figure 5A), suggesting a closer qualitative relationship of the juxtavascular c22 cluster 
cells to their perivascular counterparts. Together, these results indicate higher activation of CD8 T cells in c22 associated with the vasculature and microglial nodules but also differences in the expression of specific immune regulatory molecules between these anatomic sites.

\section{Pervasive inflammatory effect of microglial nodules}

Differential $\mathrm{T}$ cell activation patterns can be influenced by antigens or the inflammatory microenvironment. Since immune infiltration was found throughout our cohort of COVID-19 patients (microgliosis: $80 \%$ ), but microglial nodules were observed in only $28 \%$ of COVID-19 cases, we wondered if the presence of microglial nodules might orchestrate more severe neuroinflammation. This analysis was influenced by our observation of a higher antigenpresentation capacity of CD8 T cell cluster c22 expressing HLA-DR, but also higher HLA-DR expression by CD4 T cell cluster c29 and microglial cluster c7 in the nodules, indicating strong inflammation (Figure 5B). We thus analyzed the immune activation patterns of key immune cells in the different compartments depending on presence or absence of microglial nodules (Figure 5C, Supplementary Figure 1E). Of note, analysis of CD8 T cells cluster c22 revealed higher PD1 expression in the parenchyma and higher PD-1 and CD39 co-expression in the juxtavascular and parenchymal compartment in patients with microglial nodules. Together, these data clearly illustrate a pervasive pro-inflammatory effect linked to the presence of microglial nodules across all brain compartments. We next wondered if a similar immune compartmentalization also affected other immune clusters. Indeed, as shown in Figures 5B,D and Supplementary Figure 1F we observed a similar induction of HLA-DR on CD4 T cell cluster c29 or microglial cluster c7 and c15 in microglial nodules. Similar to the CD8 T cell c22, these changes were associated with a differential up-regulation of additional co-regulatory immune checkpoints, such as PD-L1, CD39 or CD33 (Figure 5D, Supplementary Figure 1F). Notably, co-expression of immune checkpoints PD-1 and PD-L1 on adjacent immune cells suggests active modulation of brain immunity through 
263 the PD-1:PD-L1 pathway (Supplementary Figure 1G). Fitting to these observations, patients 264 with microglial nodules tended to have higher numbers of APP deposits (Supplementary Figure 265 1H), suggesting a link between immune activation and axonal damage. These results 266 demonstrate significant differences in the anatomic immune regulation in COVID-19 affected 267 brains and point towards a specific role for microglial nodules and CD8 T cell-microglia crosstalk 268 in orchestrating the brain immune responses. Expression of multiple immune checkpoints by 269 these immune populations indicates potential for therapeutic manipulation. 
271 Here, by performing highly multiplexed spatial analysis of the cellular composition and

272 immunological phenotype of CNS tissue from patients that died with COVID-19, we identified a

273 profound immune activation dominated by specific CD8 T cell clusters affecting (1) the

274 vasculature and (2) significant CD8 T cell - microglial crosstalk in the parenchyma that frequently

275 manifests in the formation of microglial nodules. Remarkably, the degree of neuroinflammation

276 was variable but major changes in microglial immune activation were observed in about $80 \%$ of

277 patients. Importantly, this immune infiltration was linked to axonal damage, highlighting significant

278 severe neuroinflammation in COVID-19 patients that concomitantly affects neuronal populations

279 in the brain stem region, which might explain the high prevalence of transitory and long-lasting 280 neurological comorbidities in SARS-CoV-2 infection. These results underline the need for 281 therapeutic interventions aimed at maintaining neuronal homeostasis in SARS-CoV-2 infection.

283 Key findings of our study are the broad immune activation and anatomic compartmentalization of 284 the altered brain immune responses in COVID-19 patients. Briefly, perivascular immune activation 285 and infiltration by distinct CD8 and CD4 T cell clusters and expansion of disease-associated 286 clusters of perivascular macrophages, pericytes and microglial cells was a dominant feature found 287 in the majority (80\%) of patients. Importantly, our analysis also revealed compartimentalized 288 changes in brain immunity since the vascular immune response pattern differed quantitatively and 289 qualitatively from the parenchymal and microglial nodule immune response. Indeed, presence of 290 a moderate or severe degree of microgliosis and microglial nodules were observed in $48 \%$ and $29128 \%$ of patients, and were linked to CD8 T cell infiltration and high activation. Of note, these 292 pathologic features of microgliosis and microglial nodule are linked to increasing and pervasive 293 immune activation. In particular, cellular analysis in microglial nodules revealed high HLA-DR 294 expression and significant T cell-microglial crosstalk that occurred together with significantly 
higher immune activation in the rest of the tissue in these patients, including the perivascular compartment. This broad immune activation was linked to significant axonal damage observed in 297 the patient cohort with trends to more axonal damage in patients with microglial nodules, which 298 provides a mechanistic basis for COVID-19 encephalopathy.

299 The origin and onset of perivascular inflammation, microglial activation and expansion and their 300 relationship to each other are currently unclear. One possible explanation for the perivascular immune activation is the expression of the SARS-CoV-2 receptor ACE2 found in endothelial cells. SARS-CoV-2 virus has been detected in COVID-19 brains, but anatomic fine-mapping of infection sites is lacking ${ }^{5}$. ACE2-mediated virus uptake might lead to local immune activation by adaptive immune responses reacting to viral antigen expressed in the vascular compartment. Alternatively, dysregulated systemic cytokine responses reported in many COVID-19 patients might contribute to the perivascular phenotype ${ }^{20}$. A possible sequence of events compatible with our data could be that perivascular inflammation due to antigen recognition breaches the blood-brain barrier and permits adaptive immune cell infiltration into the brain parenchyma. However, another potential mechanism could be linked to the uptake of SARS-CoV-2 via olfactory nerves described in mice ${ }^{21}$ and fitting to the anosmia reported in the majority of patients. Uptake via the olfactory nerves could lead to viral antigen presented in the brain parenchyma in the absence of a compromised blood-brain barrier. This mechanism might cause primary activation of brain resident immunity, including microglial clusters, initially independent of adaptive immunity. While temporal or

314 mechanistic relationships could not be established in the cross-sectional post-mortem cohort 315 analyzed here, our work highlights detailed anatomic immune profiling as an important disease 316 feature required to understand COVID-19 encephalopathy.

317 In our study, we further tested for the expression of multiple targetable immune checkpoints, 318 against which therapeutic agents have been tested in clinical trials. These targets include PD-1, 319 PD-L1, CD38, CD39, Tim-3 and TIGIT. The expression of multiple immune checkpoints, including 
PD-1, on CD8 and CD4 T cells suggests that these cells can be modulated through these

321 pathways. In particular, the PD-1/PD-L1 pathway is of interest, as high expression of PD-1 on

322 CD8 T cell cluster cells and PD-L1 on closely interacting microglial clusters was observed.

323 However, it remains unclear if boosting adaptive immunity by checkpoint blockade antibodies may

324 benefit disease outcomes, as even higher T cell activation would be predicted. Indeed, the up-

325 regulation of these immune checkpoints could potentially explain the limited necrosis observed in

326 the tissues analyzed, despite the presence of CD8 effector T cells armed with lytic granula. It is

327 therefore possible that brief interruption of inhibitory signaling might boost the $\mathrm{T}$ cell immune

328 response to eliminate viral antigen in the brain. Nevertheless, such a scenario would require the

329 boosted immune response to eliminate viral antigen quickly before causing significant and

330 widespread immune pathology. There is precedent that such an approach can be effective, as

331 illustrated by the successes in treating JC-virus-associated progressive multifocal

332 leukencephalopathy with PD-1 blockade approaches ${ }^{22}$. Additional investigations of the role of

333 SARS-CoV-2 neurotropism and immunoregulation will be required to better delineate the

334 relationship between virus, antigen, neuroinflammation and immunomodulation.

335 Microglial cells fulfill many functions in brain immunity and homeostasis, and adapt quickly to 336 different challenges and disease settings. Recently, significant microglial heterogeneity during 337 development and has been highlighted by single cell expression techniques based on RNA 338 expression profiling in murine models ${ }^{23}$. Here, in order to understand the broad and 339 heterogeneous nature of the human brain immune responses during SARS-CoV-2 infection, we 340 developed an integrated IMC method that allowed the quantification of 38 immune markers and 341 included key microglial markers together with markers against major innate and adaptive immune 342 cell populations to determine the spatial brain immune response with unprecedented detail. 343 Cellular segmentation revealed significant heterogeneity in brain-specific immune populations in 344 controls and COVID-19 patients, which identified several subsets of microglial cell populations 
and homeostatic innate immunity clusters. Importantly the heterogeneity and composition of the 7 newly identified microglial clusters in our study was significantly changed in COVID-19 brains

347 towards more activated microglial clusters. In particular, two microglial clusters (clusters c7 and $348 \mathrm{c} 15)$ emerged that were significantly linked to the formation of microglial nodules, i.e. densely 349 packed areas with high microglial content that can be approximated using a novel microglial 350 nodule index. These microglial clusters displayed high immune activation and expression of 351 microglial functional markers. Importantly, in microglial nodules, they interacted closely with CD8 352 and CD4 T cells. This interaction seemed to have a pervasive effect on the global brain immune 353 response as significantly higher immune activation was observed in distant anatomic compartments in patients with microglial nodules and suggests that the microglial nodules serve as orchestrating immune hubs not unlike tertiary lymphoid structures in other organs. Of note, the broad immune infiltration in COVID-19 brains was linked to an expense of a likely homeostatic microglial cluster with high expression of CD68, which appeared to interact with a GzmB+ CD3 NK-cell-like immune population. While homeostatic microglial-NK cell relationships have been suggested by some studies ${ }^{24,25}$, evidence for a role of such spatially interacting clusters in human disease was lacking. Moreover, it remains to be clarified if the reduction of microglial homeostatic clusters and emergence of activated inflammatory microglial clusters can be explained by a potential activation and expansion of these microglial clusters. Nevertheless, while the immune profiling in this study focused on the pro-inflammatory changes associated with immune infiltration in COVID-19 brains, the observed dysbalancing of homeostatic brain immunity during COVID-19 suggests that strategies fostering homeostatic immune cluster interactions could also represent interesting therapeutic approaches to ameliorate immune activation in the brain.

367 The broad neuroinflammation observed by deep spatial immune analysis in this study points to a 368 profound CNS manifestation of COVID-19. In our large prospectively collected post-mortem 369 cohort, $80 \%$ of patients displayed significant microglial alterations, $68 \%$ of patients had notable 
370 parenchymal CD8 infiltration and $28 \%$ of patients had the most severe phenotype with profound

371 microglial nodule formation. Moreover, axonal degeneration was typically found in the medulla of

372 COVID-19 patients, but this neuropathology occurred in the absence of broad necrosis. The 373 immunological and clinically relevant alterations observed in our work fit to the high rate of 374 neurologic symptoms during COVID-194. However, these results were unexpected in light of 375 earlier assessments that mainly reported hypoxic changes and thromboembolic events and did 376 not report significant encephalitis ${ }^{9}$. Of note, only $16 \%$ of patients in our cohort had 377 thromboembolic events that also were localized to distant brain regions, suggesting that the 378 profound neuroinflammation observed in the brain stem and the thromboembolic events are 379 unrelated. The high prevalence of neuroinflammation during COVID-19 observed in our work 380 highlights the need for better strategies targeting or preventing this inflammatory encephalopathy.

381 Our study clearly underlines the relevance of high-parametric deeply resolved spatial analysis of 382 brain immunity that is able to comprehensively dissect brain immune populations, such as distinct 383 microglial clusters and multiple brain compartments in order to understand the immunological and 384 pathophysiological changes during COVID-19. The identification of disease-linked CD8 T cell385 microglial cross-talk that occurred in specific anatomic compartments and between defined 386 cellular clusters that involve multiple immune checkpoints highlights possible cellular and 387 molecular targets for therapeutic intervention. 


\section{ACKNOWLEDGMENTS}

389 This project was supported by grants from the Deutsche Forschungsgemeinschaft (DFG, German 390 Research Foundation) (SFB 992, SFB1160, SFB/TRR167, SFB/TRR179, German Excellency strategy 391 CIBSS - EXC-2189- Project ID390939984) and special research funds from the Ministry for Science, 392 Research and Art of Baden-Wuerttemberg dedicated to "COVID-19 research" and "Neuroinflammation". 393 B.B. was further supported by DFG grant BE-5496/5-1 and M.P. was further supported by the Sobek 394 Foundation, the Ernst-Jung Foundation, the Reinhart-Koselleck-Grant and Gottfried Wilhelm Leibniz-Prize.

395 H.E.M. was supported by DFG ME-3644/5-1. Graphical visualizations including the graphical abstract were 396 created with BioRender.com. We thank E. Barleon, M. Ditter and S. Hirner for excellent technical 397 assistance. 


\section{METHODS}

Ethics approvals: The analyses were performed with the approval of the Institutional Review Boards (Ethic Committee of the Albert-Ludwigs-University, Freiburg: 322/20, 10008/09; Ethics Committee of the Hamburg Chamber of Physicians: WF-051/20, PV7311). The study was performed in agreement with the principles expressed in the Declaration of Helsinki (2013).

Specimen collection: Formaldehyde-fixed paraffin-embedded sections of the upper medulla oblongata of 25 COVID-19 patients that had been tested positive for SARS-CoV-2 were obtained from autopsies that underwent routine neuropathological workup performed at the Institute of Legal Medicine of the University

406 Medical-Center of Hamburg-Eppendorf. Five aged-matched control tissues of the same location were obtained from autopsies performed at the Department of Neuropathology, Freiburg prior to the pandemic and selected due to the causes of death of the patients being unrelated to neurological or infectious 409 diseases.

Clinical data: Patient clinical data is summarized in Table 1.

411 Immunohistochemistry: For initial deparaffinization, slides were incubated at $80^{\circ} \mathrm{C}$ for one hour, then 412 deparaffinized in Xylene and in EnVision FLEX Target Retrieval Solution High pH cooking buffer for 20 413 minutes (DAKO, cat. \# K8000). Endogenous tissue peroxidase was blocked in $3 \%$ hydrogen peroxidase for 41410 minutes (Merck Millipore, cat. no 1.08597.1000). Samples were blocked with $10 \%$ normal goat serum 415 (NGS, BioScience, cat. \# 0060-01) plus 1\% Triton X 100 (Roth, cat. \# 3051.4) in TRIS buffer (EnVision Flex 416 Wash Buffer, Dako, cat. \# DM831) for one hour. Double-immunolabelling for lba1 and CD8 was performed 417 sequentially: the sections were first incubated with lba1 antibody (abcam, ab178846, 1:1000) in 10\% NGS $4181 \%$, Triton $X 100$ in TRIS buffer overnight at $+4^{\circ} \mathrm{C}$. After washing three times with TRIS buffer, sections 419 were incubated with a secondary antibody (goat anti-rabbit, Southern BioScience, 1:300, cat. \# 4050-08) 420 in $10 \%$ normal goat serum, $1 \%$ Triton X 100 in TRIS buffer for 45 minutes at RT. Three more washing steps 421 were performed before incubating the sections with $0.1 \%$ streptavidin peroxidase (BioScience, cat. \# 7100 422 05) in TRIS buffer for 45 minutes at RT. Specimens were rinsed three times with TRIS buffer. Next, slides 423 were incubated with DAB solution: 1 drop EnVision Flex DAB Chromogen (Dako, cat. \# DM827) per $1 \mathrm{ml}$ 424 EnVision Flex Substrate Buffer (Dako, cat. \# DM823). The incubation time was determined by continuous 
monitoring of the staining progress using a light microscope. Thereafter, the procedure was repeated for the immunohistochemical reaction for CD8 using a goat anti-mouse secondary antibody (Southern BioScience, 1:200, cat. \# 1031-08) and 0.1\% streptavidin-AP (BioScience, cat. \# 7100-04). Permanent Red (Monosan Permanent AP-Red Kit, Monosan, cat. \# MON-APP185) served as chromogen. Finally, the slides were counterstained with Gill's Hematoxylin solution (1.05175.0500, MerckMillipore). Coverslips were mounted with Kaisers Glycerin-Gelatine (Roth, cat. \# 6474.1). Images were taken with a 20x objective (Keyence, BioRevo). The immunohistochemical reactions for APP (Merck, cat. \# MAB348) and ACE2 (Novus Biologicals cat. \# NBP2-80035) were performed using the EnVision FLEX Target Retrieval Solution Low pH cooking buffer and the EnVision FLEX system (DAKO, \# K8000). Slides were counterstained with Gill's Hematoxylin solution (1.05175.0500, MerckMillipore). Coverslips were mounted with xylene-based Vitro-Clud mounting medium (R. Langenbrinck, cat. \# 04-0001). APP-positive deposits were counted manually using a light microscope (Olympus, BX41). Representative pictures were acquired with a 20x and 40x objective (Leica, DFC450).

IMC antibody panel: An IMC panel was designed including markers for the adaptive and innate immune system as well as brain-specific cell populations (Table 2). Metal-labeled antibodies were either obtained pre-conjugated (Fluidigm) or labeled in-house by conjugating purified antibodies to lanthanide metals using the Maxpar X8 antibody labelling kit (Fluidigm) according to the manufacturer's instructions. 89-Yttrium (III) nitrate tetrahydrate (Sigma Aldrich, cat. \# 217239-10G) and 157-Gadolinium (III) chloride (Trace Sciences Int.) were diluted in L-buffer to a $1 \mathrm{M}$ stock solution and further diluted to a $50 \mu \mathrm{M}$ working solution for subsequent antibody labelling with the Maxpar X8 labelling kit. Metal-conjugated antibodies were titrated and validated on brain, liver and tonsil tissue.

Sample preparation and staining for $I M C$ : Tissue sections were baked at $60^{\circ} \mathrm{C}$ for one hour, dewaxed in Xylene twice for 15 minutes and rehydrated in descending grades of ethanol (100\% - 100\% - 95\% - 80\%) for five minutes each, then rinsed in TBS $(\mathrm{pH} \mathrm{7.6)}$ for 10 minutes. Epitope retrieval was performed in a decloaking chamber (Biocare Medical) with EnVision FLEX Target Retrieval Solution High pH for 30 minutes at $95^{\circ} \mathrm{C}$. Slides were cooled down to room temperature for 20 minutes inside the buffer and 10 minutes in TBS. Tissue sections were encircled with a PAP pen (ImmEdge, Vector laboratories, cat. \# H- 
452 4000) and blocked for 45 minutes at room temperature using SuperBlock (TBS) Blocking Buffer 453 (ThermoFisher Scientific, cat. \# 37581). Afterwards, the sections were stained with a mix of metal-labeled 454 primary antibodies diluted in TBS with $0.05 \% \mathrm{BSA}$ and incubated at $4^{\circ} \mathrm{C}$ overnight. Slides were rinsed in 455 TBS-T twice (TBS supplemented with 0.2\% Tween-20) and twice in TBS for 5 minutes each. Tissue 456 sections were then stained with 1:2000 Iridium Cell-ID intercalator (500 $\mu \mathrm{M}$, Fluidigm cat. \# 201192B) in 457 TBS for 30 minutes at room temperature. Slides were rinsed three times for 5 minutes in TBS, dipped in $458 \mathrm{ddH} 2 \mathrm{O}$ for 5 seconds and air-dried. Slides were stored at room temperature until image acquisition.

459 Image acquisition: One $1500 \mu \mathrm{m}^{2}$ image per sample was acquired using a Hyperion Imaging System 460 (Fluidigm). Briefly, tuning of the instrument was performed according to the manufacturer's instructions. 461 Regions of interest were determined using an Iba1 and CD8a double immunohistochemistry staining. Laser 462 power was set to three and tissue sections were laser-ablated spot-by-spot at $200 \mathrm{~Hz}$ resulting in a pixel 463 size/resolution of $1 \mu \mathrm{m}^{2}$. Preprocessing of the raw data was conducted using the CyTOF software v7.0 464 (Fluidigm). Image acquisition control was performed using MCD Viewer v1.0.560.6 (Fluidigm).

465 Data processing: MCD files containing the raw data were converted into tiff files and segmented into single 466 cells using an unbiased, supervised analysis pipeline adapted from 467 https://github.com/BodenmillerGroup/ImcSegmentationPipeline and processed as reported previously ${ }^{17}$. 468 Briefly, nucleated cells were segmented using a combination of CellProfiler v3.1.9 and llastik v1.3.3. Pixels 469 were classified into nuclei, cytoplasm and background using llastik. A probability map for the three 470 classifications was generated and used to create a cell mask with CellProfiler. For combination with a 471 subsequent lba1 mask, lba1 signal was classified as background. A second cell mask was then created in 472 CellProfiler using Iba1 expression of the images. Both masks were combined in CellProfiler using the "AND" 473 function in ImageMath.

474 Data folders containing tiff images of the 38 markers, seven background channels and the combined cell 475 mask were loaded into histoCAT v1.76 (https://github.com/BodenmillerGroup/histoCAT) and mean marker 476 intensity of pixels and spatial features of segmented cells were calculated. 
Manual cell count: $\mathrm{CD}^{+}{ }^{+} \mathrm{CD} 8^{+}, \mathrm{CD}^{+}{ }^{+} \mathrm{CD} 4^{+}, \mathrm{CD} 3-\mathrm{GmBB}^{+}, \mathrm{CD}^{2} 0^{+}$, parenchymal lba1+ microglia cells and $\mathrm{CD}_{163^{+}}$perivascular macrophages were visualized in MCD viewer and counted manually by two independent blinded scientists.

Data transformation and normalization: The presented data was normalized to $99^{\text {th }}$ percentile for Phenograph clustering (in histoCAT) Fig. 2A und 2B. For opt-SNE analysis presented in Fig. 2A, 2C and 2E, heatmap visualization and gating in Omiq.ai (Santa Clara, CA), all channels used for further analysis were arcsinh transformed with a cofactor of 0.2. In heatmaps (Fig. 2B, 3D and 3E) z-scored cluster means are depicted.

High-dimensional analysis: Phenograph clustering algorithm implemented in histoCAT (v1.76) was used to identify even rare clusters of cells with distinct marker expressions using the following settings: Nearest neighbors $=15$, random seed $=2$ and clustering was performed based on data from channels containing lineage and activation markers: CD103, CD163, CD204, CD20, CD33, CD34, CD39, CD3, CD45RO, CD45, CD4, CD68, CD8a, Collagen I, Eomes, GFAP, GzmB, HLA-DR, Iba1, Ki-67, NeuN, PD-1, PD-L1, Tim-3. Segmentation events with an area of $<20$ pixels were excluded. Single-cell cluster data was integrated with expression data and exported for further analysis to FlowJo (TreeStar, Inc.) and Omiq (Omiq, Inc.). Analysis was performed on Phenograph clusters containing more than 100 cells in the total dataset consisting of total $n=96,882$ cells.

$t$-SNE analysis: Visualization of the global single-cell landscape was performed using Opt-SNE in Omiq based on channels (CD103, CD163, CD204, CD20, CD33, CD34, CD39, CD3, CD45RO, CD45, CD4, CD68, CD8a, Collagen I, Eomes, GFAP, GzmB, HLA-DR, Iba1, Ki-67, NeuN, PD-1, PD-L1, Tim-3), random seed 3800, max iterations 1000, opt-SNE end 5000, perplexity 30, theta 0.5 and verbosity 25 . Subsequently, Phenograph clusters identified as immune cells were visualized using opt-SNE calculated on immune cell markers (CD103, CD163, CD204, CD20, CD33, CD39, CD3, CD45RO, CD45, CD4, CD68, CD8a, Eomes, GzmB, HLA-DR, Iba1, Ki-67, PD-1, PD-L1, Tim-3, TOX), random seed 691, max iterations 1000, opt-SNE end 5000, perplexity 30, theta 0.5 and verbosity 25.

Heatmaps: Signal intensity of marker expression in Phenograph clusters was determined in Omiq and visualized in a heatmap in $\mathrm{R}$ studio v4.0.2 Pheatmap package. 
Cluster compositions (stacked bar graph): Clusters were gated on patients and counts were exported from Omiq. The cluster composition between patient groups was visualized using the ggplot2 stacked bar graph

506 function in R studio.

507 Correlation heatmaps: Linear regression analysis of Phenograph cluster frequencies in COVID-19 samples was performed pairwise across all Phenograph clusters (JMP 12.2.0, SAS Institute, Inc). P values for individual cluster pairs were determined and summarized in a cluster matrix. $\mathrm{P}$ values were then visualized as a heatmap using $R$ v4.0.2 Pheatmap package.

511 Neighborhood analysis: Spatial cellular interaction patterns were tested by neighborhood analysis as 512 implemented in histoCAT, v1.76. Briefly, it was examined per image whether a cell of a certain Phenograph 513 cluster was located significantly $(p=0.05)$ more frequently in close proximity (4 pixel) to a cell of another 514 cell cluster than expected by a random distribution of cells. The results for each cluster pair were 515 summarized and visualized in a heatmap using R v4.0.2 Pheatmap package.

516 Vascular proximity map: FIJI, a distribution of ImageJ, v1.52p, was used for the calculation of the proximity 517 to the closest Collagen-I-positive structure. Background and noise were reduced by excluding extremely 518 weak signal in the 169Tm-Collagen-I imaging mass cytometry channel (threshold 1.65) and the median 519 filter (radius 0.5 pixels). The values for all 2.25 million pixels per image were obtained and plotted with 520 ggplot2 function in R studio.

521 Microglia nodule index map: The calculation of the microglia nodule index map was performed in a similar 522 way to the vascular proximity map. The starting point was the signal in the 141Pr-lba1 channel. The 141Pr523 Iba1-positive pixels were measured in a radius of $15 \mu \mathrm{m}$ and put in relation to the total area. The values 524 were calculated for all 2.25 million pixels in the image. The map was again visualized using ggplot2 in R 525 studio.

526 Microglia nodule and microgliosis quantification and pie chart: The composition of the cohort was visualized 527 based on the criteria of microgliosis (moderate microgliosis with $>80$ microglia cells per $\mathrm{mm}^{2}$ and severe 528 microgliosis $>160$ cells per $\mathrm{mm}^{2}$ ) and the presence or absence of microglia nodules. 
529 CNS compartments: Distances to vasculature inferred by distance to nearest collagen-I expressing pixel 530 and microglia nodule index were used to identify 4 compartments: Microglia nodules (nodule index $\geq 0.5$ ), 531 perivascular (nodule index $<0.5$, distance to collagen- $\mathrm{I}<5 \mu \mathrm{m}$ ), juxtavascular (nodule index $<0.5$, distance 532 to collagen-I $\geq 5 \mu \mathrm{m}$ and $\leq 20 \mu \mathrm{m}$ ), parenchymal (nodule index $<0.5$, distance to collagen-I $>20 \mu \mathrm{m}$ ).

533 Statistical analysis: Statistical analyses were performed using GraphPad Prism 8 (GraphPad Software, 534 Inc.). Differences in cell counts of clusters and cell populations as well as APP density between COVID-19 535 and control patients in Figures 1D, 1E, 3A and 3F were analyzed by t-tests with Welch's correction. 536 Frequencies of cell populations between different CNS compartments in figures 4E and 4F were analyzed 537 by Mann-Whitney-U tests. If not indicated otherwise in figure legends, throughout the manuscript * indicates $538 \mathrm{p}<0.05,{ }^{* *} \mathrm{p}<0.01$ and ${ }^{* * *} \mathrm{p}<0.001,{ }^{* * *} \mathrm{p}<0.0001$ 


\section{Figure 1: Highly multiplexed imaging mass cytometry analysis of COVID-19 brains.}

541

542

543

544

545

546

547

548

549

550

551

552

553

554

555

556

557

558

559

560

561

562

563

564

565

566

567

568

569

570

571

572

573

574

575

576

577

A: Graphical illustration of the experimental workflow. Medulla oblongata tissue slices from autopsies of COVID-19 patients $(n=25)$ and controls $(n=5)$ underwent parallel conventional immunohistochemistry and high-dimensional imaging mass cytometry (IMC) with 38 markers.

B: Exemplary immunohistochemistry (left) and corresponding IMC visualization (right) of the upper medulla tissue of a COVID-19 patient. The area of interest is magnified in the right upper corner. Scale bars indicate $100 \mu \mathrm{m}$ and $50 \mu \mathrm{m}$, respectively. Left: Double-chromogenic immunohistochemical reaction for lba1 (brown) and CD8 (pink), counterstained with haematoxylin (blue). Arrows indicate CD8a+ T cells. Right: Image overlay visualization of Iba1 (red), CD8 (green), collagen-I (light blue), CD163 (yellow) and histone H3 (blue) IMC data out of the 38-marker dataset.

C: Visualization of the indicated marker expression data from channels shown exemplarily for one area of interest as indicated in B). The scale bar represents $50 \mu \mathrm{m}$.

D: Manual counts of microglial cells, perivascular macrophages, CD4, CD8 T cells, B cells and NK cells was performed on the IMC dataset and compared between controls (blue) and COVID-19 patients (green). Data are shown as bar graphs \pm SEM.

E: Robust axonal damage in COVID-19 brains: immunohistochemical reaction for APP (brown) in the upper medulla of control and COVID-19 patients. Counterstaining with hematoxylin (blue). Scale bars indicate $100 \mu \mathrm{m}$ and $10 \mu \mathrm{m}$ in the inserts, respectively. The quantification of APP-positive deposits in the whole section is shown next to the histological images. Data are shown as bar graphs \pm SEM.

Significant differences were determined by unpaired $t$ tests with Welch's correction $(D, E)$. In $D$ all controls were negative for manual $\mathrm{B}$ cell counts, therefore a one sample $t$ test was used here.

\section{Figure 2: Molecular and cellular census of neuroinflammation during SARS-CoV-2 infection.}

A: Segmentation of the highly multiplexed IMC images into cellular masks representing single cells was performed on all patients and controls using supervised machine learning. Single-cell data was extracted and cells were clustered using Phenograph and visualized on a $t$-SNE map.

B: Heatmap displaying mean marker expression (shown as z-score) of the Phenograph clusters. Clusters were annotated using the expression and spatial distribution of the cells.

C: The neuroimmune landscape of SARS-CoV-2 infected and non-infected patients was visualized by $t$ SNE based on the immunological clusters determined in B.

D: The immune cell cluster composition was assessed between the two groups. Stacked bar charts indicate mean counts per group.

E: The brain immune map from C is plotted according to cellular origin: cells from COVID-19 patients are depicted in blue; cells from controls are shown in orange.

Figure 3: Profiling of the CNS immune response during COVID-19 indicates a localized and orchestrated adaptive immune infiltration. 
A: High-dimensional annotated cells were compared across patients and controls. Typical cell clusters with significantly different abundances are displayed. Means are presented as bar graphs, error bars are indicating \pm SEM.

B: Pairwise linear regression analysis between all cluster frequencies in the brains of COVID-19 patients was conducted and $p$-values of individual cluster pairs were visualized in a heatmap.

C: Violin plot indicating the distance of $\mathrm{CD}^{+}$cells to the nearest vessel (collagen-I-positive) structure in COVID-19 patients. A random distribution is shown for comparison. The $25^{\text {th }}, 50^{\text {th }}$ and $75^{\text {th }}$ percentiles are depicted. The CD8 T cell distribution is further visualized by an overlaid scattered dot plot, showing all CD8+ cells and the corresponding distance to the closest vessel structure in 25 COVID-19 patients. Each dot represents one cell.

D: Spatial interactions between cell clusters were assessed in COVID-19 patients by permutation based neighborhood analysis and displayed as hierarchically clustered heatmap of the neighborhood interaction (fraction of significant images) ranging from -1 (avoidance) to +1 (preferential interaction). Rows indicate the neighborhood of a cell phenotype of interest. Columns indicate the enrichment or depletion of a cell in other neighborhoods.

E: Cluster 22 spatial neighborhood interactions were determined in COVID-19 and control patients. Rows indicate the neighborhood of a cell phenotype of interest.

F: CD8 T-cell activation in the brains of COVID-19 patients. Cluster 22 cells were tested for expression of immune markers involved in T-cell activation, differentiation, exhaustion and function. Absolute cell counts were compared between patient groups. Data are presented as boxplots showing the median, IQR and upper and lower whiskers and dots represent samples.

Significant differences were determined by unpaired $t$ tests with Welch's correction (A, F).

\section{Figure 4: Spatial compartmentalization of immune cell clusters to distinct anatomical niches in the brain of COVID-19 patients.}

A: Left: Representative IMC image showing lba1 (red) and CD8a (green) expression. Middle: Microglial distribution was assessed using the accumulation of lba1 signal across a $15 \mu \mathrm{m}$ and visualized in a microglia nodule index map. Right: magnified inserts of the area of interest indicated in the left and middle panel. Scale bars indicate $100 \mu \mathrm{m}$ and $50 \mu \mathrm{m}$, respectively.

B: Vascular distance was estimated as distance to collagen-I. A proximity map displaying the distance of each pixel to the next Collagen-I-positive structure was generated and overlaid with collagen-I expression signal. Close proximity is indicated by increasing darkness. Collagen-I expression is indicated in blue.

C: Patient sections were assessed for presence of microgliosis or microglial nodules and visualized in a pie chart. There was a significant difference in the prevalence of microglial alterations (microgliosis and/or nodules) between the two groups.

D: Cluster composition of anatomical compartments. Images show examples of anatomical niches in one patient sample. Image overlay visualization of Iba1 (red), CD8a (green), collagen I (light blue), CD163 (yellow) and histone $\mathrm{H} 3$ (blue). Scale bar indicates $50 \mu \mathrm{m}$. Arrows and asterisks point to CD8 T cells located inside a vessel (perivascular), close to a vessel (juxtavascular), infiltrating into the parenchyma (parenchymal) and inside a microglial nodule (nodule compartment), respectively. Cluster composition of the four compartments was evaluated for all COVID-19 patients and displayed as stacked bar graphs of cluster frequencies per anatomical compartment. 
620 E: Enrichment of individual clusters in anatomic compartments was assessed by calculating and visualizing 621 the enrichment index (mean cluster frequency in given compartment / mean frequency in the total COVID62219 cohort).

623 Unpaired $t$ test with Welch's correction was used in B.

624 Figure 5: Assessment of immune cell activation in different anatomical compartments 625 suggests pervasive inflammatory effect of microglial nodules on distant T-cell activation 626 and immune checkpoint expression.

627 A: Analysis of CD8 T cells in different CNS compartments. Frequencies of PD $-1^{+}$, CD39+, PD $-1^{+}$CD39+, $628 \mathrm{Tim}^{+}$and Eomes ${ }^{+}$and HLA-DR ${ }^{+}$cells of cluster 22 were compared across perivascular, juxtavascular, 629 parenchymal and nodule compartments. Boxplots show the median with IQR and upper and lower whiskers.

630 B: HLA-DR expression of CD8 T cells, CD4 T cells and activated microglial cells in different CNS 631 compartments. Left: Spatial heatmap displaying HLA-DR signal intensities in segmented cells on the image. 632 Scale bar indicates $100 \mu \mathrm{m}$. Right: Frequencies of HLA-DR+ cells of cluster c22 (CD8 T cells), c29 (CD4 T 633 cells), c7 (activated microglia) and c15 (activated microglia) compared across perivascular, juxtavascular, 634 parenchymal and nodule compartments. Boxplots show the median with IQR and upper and lower whiskers.

635 C: CD8 T-cell activation in separate anatomical regions. Patients were grouped based on the presence or absence of microglial nodules in the image and the frequencies of PD-1+, CD39+, PD-1+CD39+, Eomes ${ }^{+}$ and HLA-DR+ of cluster c22 were compared between those patients in the perivascular, juxtavascular and parenchymal compartment to reveal a pervasive effect of microglial nodules on the activation of CD8 T cells. Boxplots show the median with IQR and upper and lower whiskers.

640 D: Compartment-dependent expression of activation and immunoregulatory molecules by activated 641 microglial cells. Frequencies of $\mathrm{CD}^{+} 8^{+}, \mathrm{CD}_{3}{ }^{+}, \mathrm{CD} 39^{+}, \mathrm{Tim}_{-3}{ }^{+}$and $\mathrm{PD}-\mathrm{L} 1^{+}$cells of cluster c7 were 642 compared across the four different CNS compartments. Boxplots show the median with IQR and upper and 643 lower whiskers.

644 E: Exemplary image showing PD-1 (blue) and PD-L1 (red) expression on a spatial heatmap of segmented 645 cells.

646 Mann-Whitney-U non-parametric t-tests were used in A-D. 


\section{Supplementary figure 1:}

649 A: Bar graph visualizing cell counts of Phenograph clusters. Clusters are enumerated based on abundance of a given cluster in the combined analysis of all tissue samples.

651 B: Immunohistochemical reaction for ACE2 (brown) in the upper medulla of a control patient. 652 Counterstaining with haematoxylin (blue). An ACE2-positive structure, histopathologically corresponding to 653 a vessel, is indicated with an arrow. The scale bar represents $50 \mu \mathrm{m}$.

654 C: Cluster 22 spatial neighborhood interactions were determined in COVID-19 patients and controls. Spatial 655 interactions of single c22 cells in other neighborhoods are shown.

656 D: Image overlay visualization of Iba1 (red), CD8a (green), collagen I (light blue), CD163 (yellow) and histone $\mathrm{H} 3$ (blue). Scale bar indicates $100 \mu \mathrm{m}$. Boxes indicate exemplary images of anatomical compartments shown in Figure 4D. Region 1: perivascular compartment, region 2: juxtavascular compartment, region 3: parenchyma, region 4: nodule compartment.

660 E: Frequency of Tim-3+ cells of cluster c22 compared between COVID-19 patients without microglial nodules (orange) and with microglial nodules (red) in the perivascular (left), juxtavascular (middle) and parenchymal (right) compartment respectively. Boxplots show the median with IQR and upper and lower whiskers.

F: Compartment-dependent expression of activation and immunoregulatory molecules by activated microglial cells. Frequencies of $\mathrm{CD}_{68}{ }^{+}, \mathrm{CD}_{3}{ }^{+}, \mathrm{CD}_{3}{ }^{+}, \mathrm{Tim}^{-} 3^{+}$and $\mathrm{PD}-\mathrm{L} 1^{+}$cells of cluster $\mathrm{c} 15$ were compared across the four different CNS compartments. Boxplots show the median with IQR and upper and 667 lower whiskers.

668 G: PD-1 and PD-L1 signal intensities in segmented cells on the image.

$669 \mathrm{H}$ : Axonal damage in controls and COVID-19 patients: data from Figure 1E is depicted according to the 670 presence or absence of microglia nodules. Data are shown as scattered dot plot. Error bars indicate SEM. 671 Each dot represents one patient.

672 Mann-Whitney-U non-parametric t-tests were used in E and F.

\section{SUPPLEMENTARY TABLES}

\section{Table 1: Cohort and clinical data}

676 Table showing patient group (COVID-19 and control), sex, age, place of death (ER: Emergency room, 677 hosp.: Hospital ward, ICU: Intensive care unit, NH: Nursing home), post-mortem interval (PMI), cause of 678 death (pe: pulmonary artery embolisms, scd: sudden cardiac death), comorbidities (Cl: cardiac 679 insufficiency, COPD: chronic obstructive pulmonary disease, DM: diabetes mellitus, IHD: ischemic heart 680 disease, RI: renal insufficiency, Tx: Transplantation) and details of the brain autopsy for each patient.

\section{Table 2: IMC brain adaptive and innate immune system panel}

683 Table listing antibody clones used for IMC staining with information on metal label, company of which the 684 antibody was obtained, catalog number and dilution at which the antibody was used. 


\section{REFERENCES}

6851 Mao, L. et al. Neurologic Manifestations of Hospitalized Patients With Coronavirus

686

687

688

689

690

691

692

693

694

695

696

697

698

699

700

701

702

703

704

705

706

707

708

709

710

711

712

713

714

715

716

717

718

719

720

721

722

723

724

725

726

727

728

729

730

731

732

Disease 2019 in Wuhan, China. JAMA Neurol, doi:10.1001/jamaneurol.2020.1127 (2020).

2 Helms, J. et al. Neurologic Features in Severe SARS-CoV-2 Infection. N Engl J Med 382, 2268-2270, doi:10.1056/NEJMc2008597 (2020).

3 Varatharaj, A. et al. Neurological and neuropsychiatric complications of COVID-19 in 153 patients: a UK-wide surveillance study. Lancet Psychiatry, doi:10.1016/S22150366(20)30287-X (2020).

4 Ellul, M. A. et al. Neurological associations of COVID-19. Lancet Neurol, doi:10.1016/S1474-4422(20)30221-0 (2020).

5 Puelles, V. G. et al. Multiorgan and Renal Tropism of SARS-CoV-2. N Engl J Med 383, 590-592, doi:10.1056/NEJMc2011400 (2020).

6 Kantonen, J. et al. Neuropathologic features of four autopsied COVID-19 patients. Brain Pathol, doi:10.1111/bpa.12889 (2020).

7 Reichard, R. R. et al. Neuropathology of COVID-19: a spectrum of vascular and acute disseminated encephalomyelitis (ADEM)-like pathology. Acta Neuropathol 140, 1-6, doi:10.1007/s00401-020-02166-2 (2020).

8 Schaller, T. et al. Postmortem Examination of Patients With COVID-19. JAMA, doi:10.1001/jama.2020.8907 (2020).

9 Solomon, I. H. et al. Neuropathological Features of Covid-19. N Engl J Med, doi:10.1056/NEJMc2019373 (2020).

10 von Weyhern, C. H., Kaufmann, I., Neff, F. \& Kremer, M. Early evidence of pronounced brain involvement in fatal COVID-19 outcomes. Lancet 395, e109, doi:10.1016/S01406736(20)31282-4 (2020).

11 Mathew, D. et al. Deep immune profiling of COVID-19 patients reveals distinct immunotypes with therapeutic implications. Science, doi:10.1126/science.abc8511 (2020).

12 Kuri-Cervantes, L. et al. Comprehensive mapping of immune perturbations associated with severe COVID-19. Sci Immunol 5, doi:10.1126/sciimmunol.abd7114 (2020).

13 Prinz, M. \& Priller, J. The role of peripheral immune cells in the CNS in steady state and disease. Nat Neurosci 20, 136-144, doi:10.1038/nn.4475 (2017).

14 Tay, T. L. et al. A new fate mapping system reveals context-dependent random or clonal expansion of microglia. Nat Neurosci 20, 793-803, doi:10.1038/nn.4547 (2017).

15 Market, M. et al. Flattening the COVID-19 Curve With Natural Killer Cell Based Immunotherapies. Front Immunol 11, 1512, doi:10.3389/fimmu.2020.01512 (2020).

16 Zheng, M. et al. Functional exhaustion of antiviral lymphocytes in COVID-19 patients. Cell Mol Immunol 17, 533-535, doi:10.1038/s41423-020-0402-2 (2020).

17 Schapiro, D. et al. histoCAT: analysis of cell phenotypes and interactions in multiplex image cytometry data. Nat Methods 14, 873-876, doi:10.1038/nmeth.4391 (2017).

18 Levine, J. H. et al. Data-Driven Phenotypic Dissection of AML Reveals Progenitor-like Cells that Correlate with Prognosis. Cell 162, 184-197, doi:10.1016/j.cell.2015.05.047 (2015).

19 Bengsch, B. et al. Epigenomic-Guided Mass Cytometry Profiling Reveals DiseaseSpecific Features of Exhausted CD8 T Cells. Immunity 48, 1029-1045 e1025, doi:10.1016/j.immuni.2018.04.026 (2018).

20 Sinha, P., Matthay, M. A. \& Calfee, C. S. Is a "Cytokine Storm" Relevant to COVID-19? JAMA Intern Med, doi:10.1001/jamainternmed.2020.3313 (2020).

21 Netland, J., Meyerholz, D. K., Moore, S., Cassell, M. \& Perlman, S. Severe acute respiratory syndrome coronavirus infection causes neuronal death in the absence of 
encephalitis in mice transgenic for human ACE2. $J$ Virol 82, 7264-7275, doi:10.1128/JVI.00737-08 (2008).

22 Cortese, I. et al. Pembrolizumab Treatment for Progressive Multifocal Leukoencephalopathy. N Engl J Med 380, 1597-1605, doi:10.1056/NEJMoa1815039 (2019).

23 Masuda, T. et al. Spatial and temporal heterogeneity of mouse and human microglia at single-cell resolution. Nature 566, 388-392, doi:10.1038/s41586-019-0924-x (2019).

24 Lunemann, A. et al. Human NK cells kill resting but not activated microglia via NKG2Dand NKp46-mediated recognition. J Immunol 181, 6170-6177, doi:10.4049/jimmunol.181.9.6170 (2008).

25 Hao, J. et al. Central nervous system (CNS)-resident natural killer cells suppress Th17 responses and CNS autoimmune pathology. J Exp Med 207, 1907-1921, doi:10.1084/jem.20092749 (2010). 

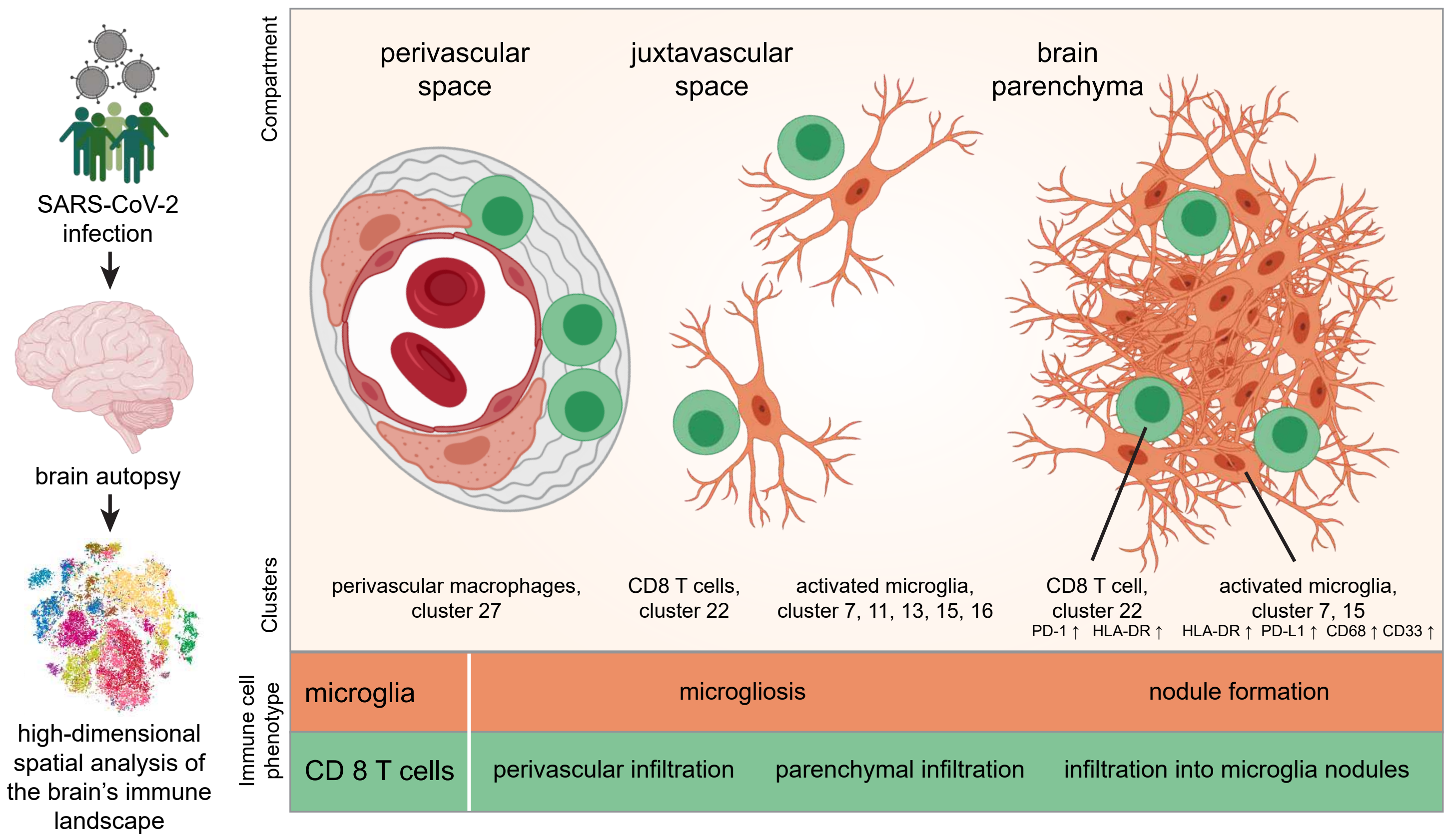

Fig. Graphical Abstract 
A 俩闬

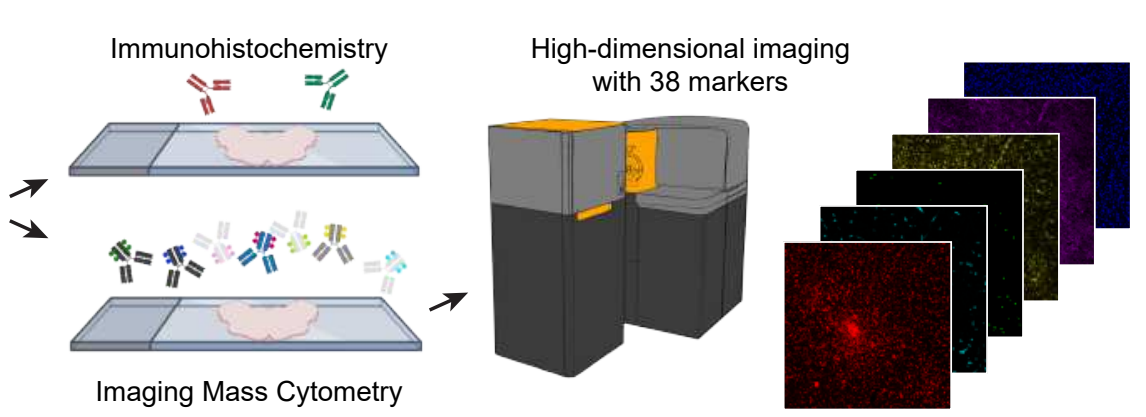

B

Immunohistochemistry

Imaging Mass Cytometry
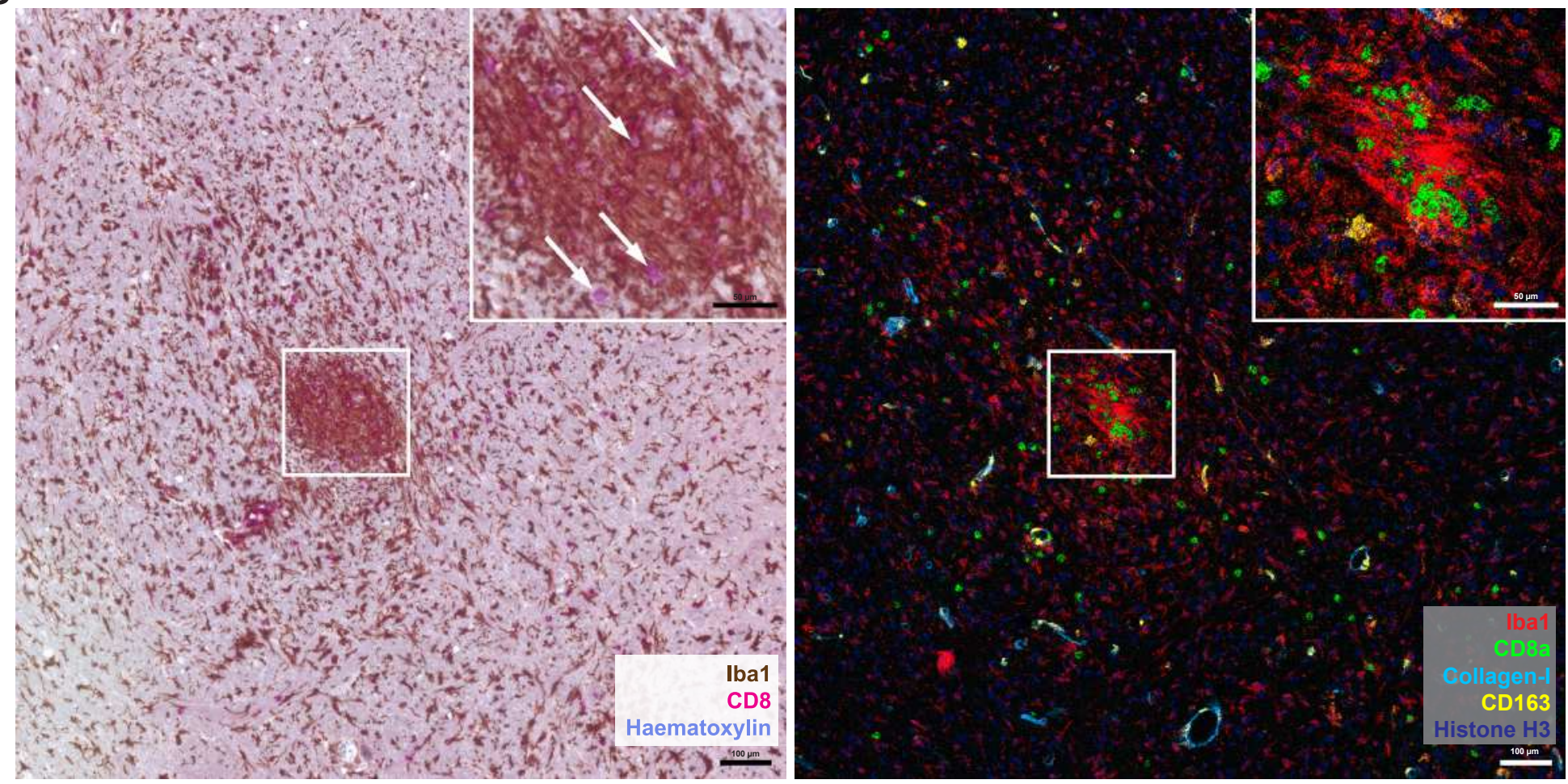

C
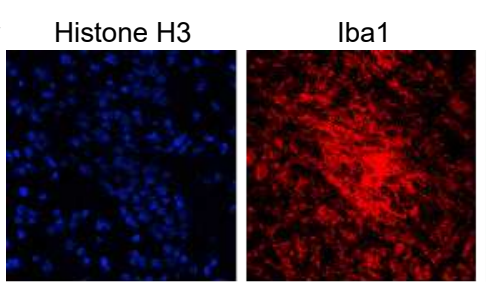

HLA-DR
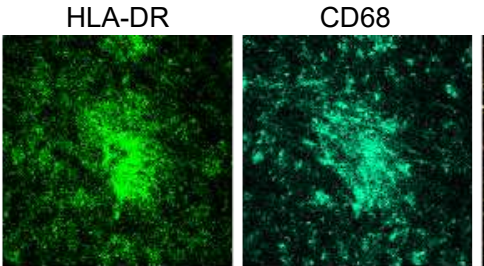

CD204

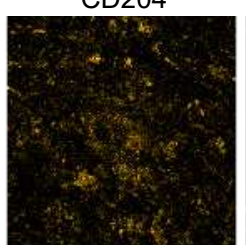

CD45RO

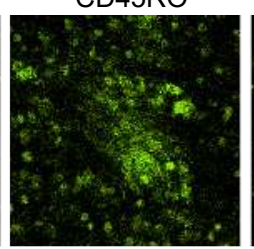

CD163

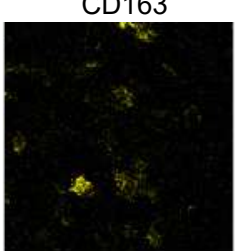

Collagen-I
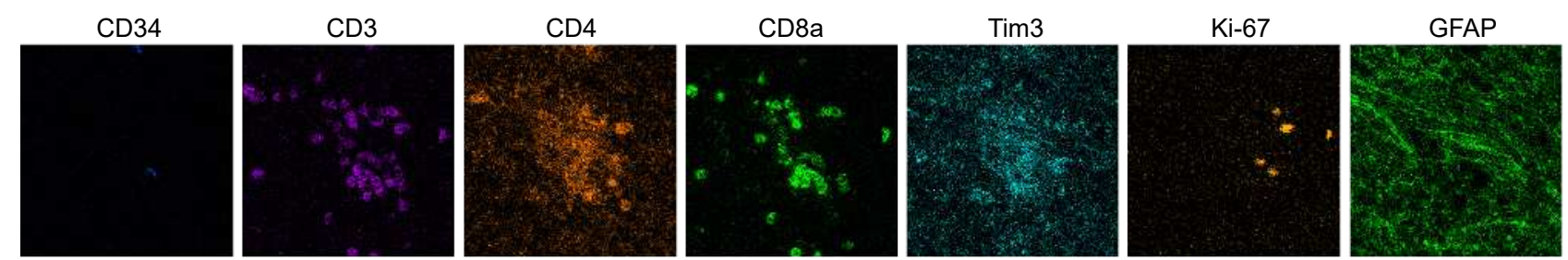

CD56
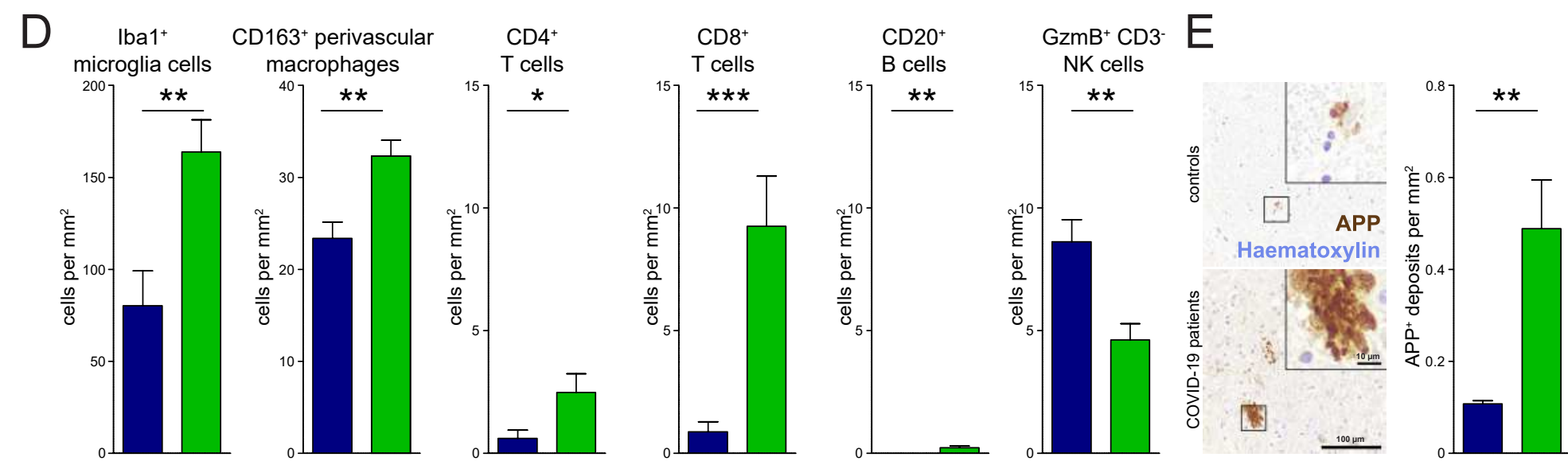

controls COVID-19 patients

Fig. 1 

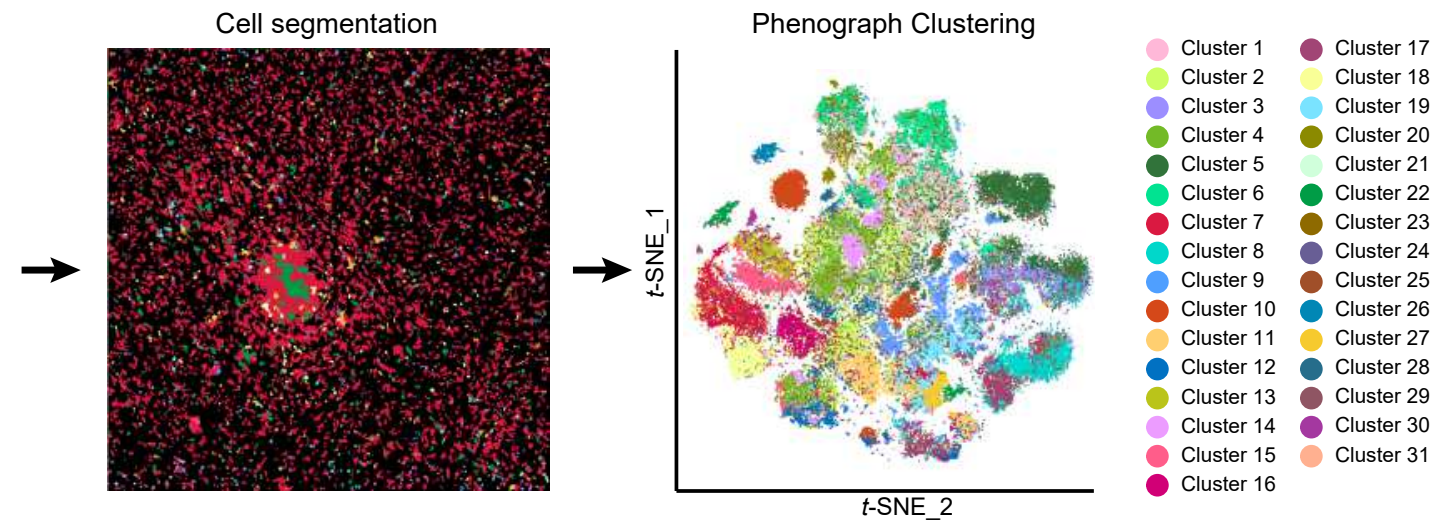

B

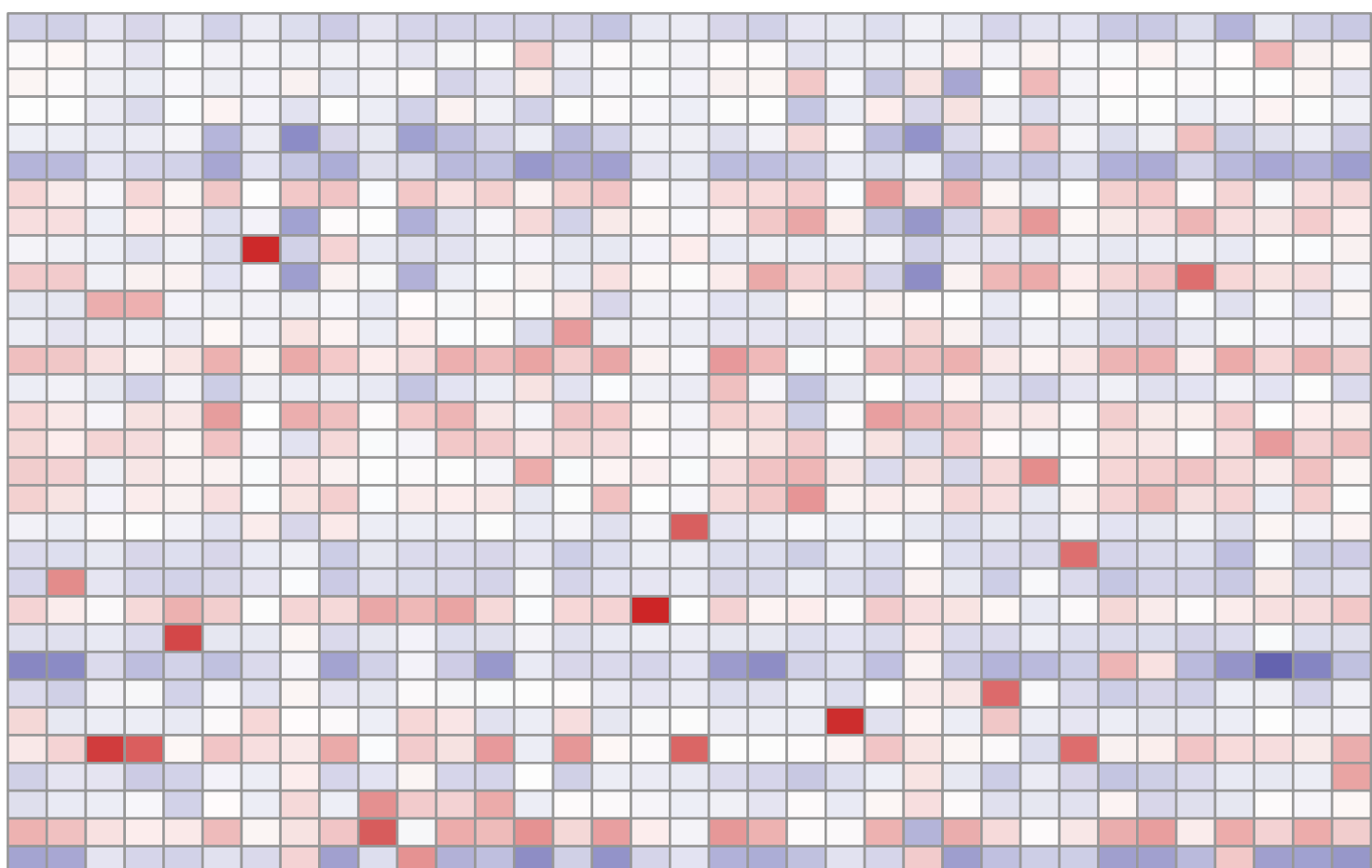

Cluster 1 Stromal cells

Cluster 2 Neurons

Cluster $3 \bigcirc$ Astrocytes

Cluster 4 Stromal cells

Cluster 5 Astrocytes

Cluster 6 Stromal cells

Cluster 7 Activated microglia

Cluster 8 Stromal cells

Cluster 9 Endothelial cells

Cluster 10 Stromal cells

Cluster 11 Activated microglia

Cluster 12 Homeostatic microglia

Cluster 13 Activated microglia

Cluster $14 \bigcirc$ Stromal cells

Cluster 15 Activated microglia

Cluster 16 Activated microglia

Cluster 17 Stromal cells

Cluster 18 Astrocytes

Cluster 19 Pericytes

Cluster 20 Neurons

Cluster 21 Stromal cells

Cluster $22 \mathrm{CD}^{+} \mathrm{T}$ cells

Cluster 23 B cells

Cluster 24 Stromal cells

Cluster 25 Proliferating cells

Cluster 26 NK cells

Cluster 27 Perivascular macrophages

Cluster 28 Stromal cells

Cluster $29 \mathrm{CD}^{+} \mathrm{T}$ cells

Cluster 30 Immune cells

Cluster $31 \bigcirc$ Other cells

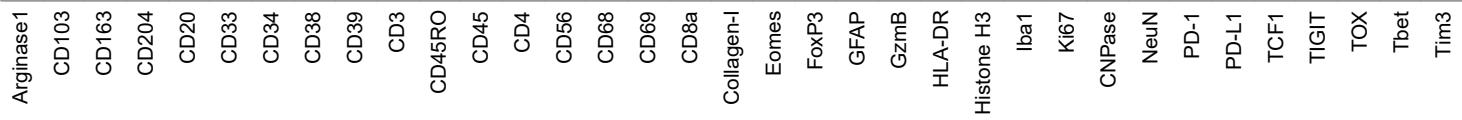

C

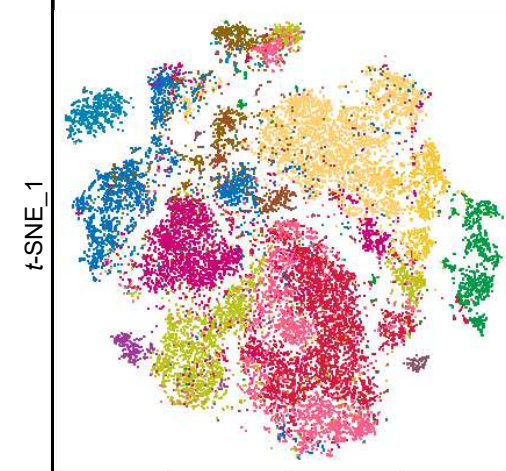

t-SNE_2
Cluster 7 Activated microglia Cluster 11 Activated microglia Cluster 12 Homeostatic microglia Cluster $13 \bigcirc$ Activated microglia Cluster $15 \bigcirc$ Activated microglia Cluster 16 Activated microglia Cluster $22 \bigcirc \mathrm{CD}^{+} \mathrm{T}$ cells Cluster 23 B cells Cluster 25 Proliferating cells Cluster 26 NK cells Cluster 27 Perivascular macrophages Cluster $29 \bigcirc \mathrm{CD}^{+} \mathrm{T}$ cells Cluster 30 Immune cells
D cluster composition

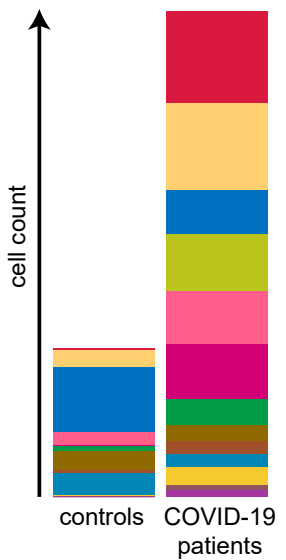

E

Cluster 7
Cluster 11
Cluster 12
Cluster 13
Cluster 15
Cluster 16
Cluster 22
Cluster 23
Cluster 25
Cluster 26
Cluster 27
Cluster 29
Cluster 30
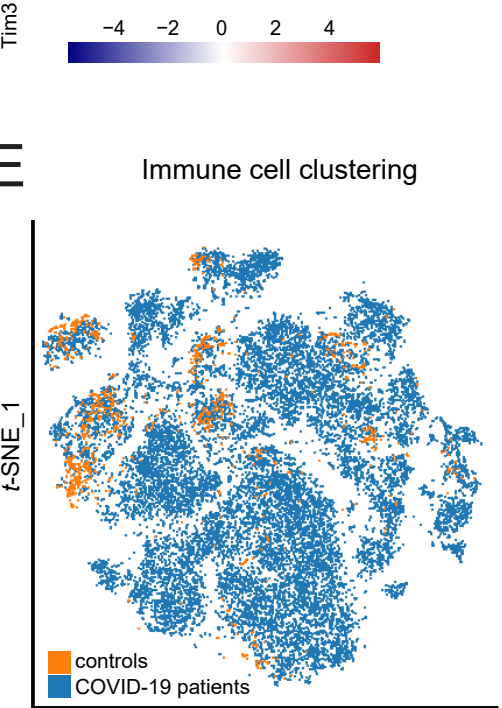

$t$-SNE_2

Fig. 2 
A Imaging Mass Cytometry
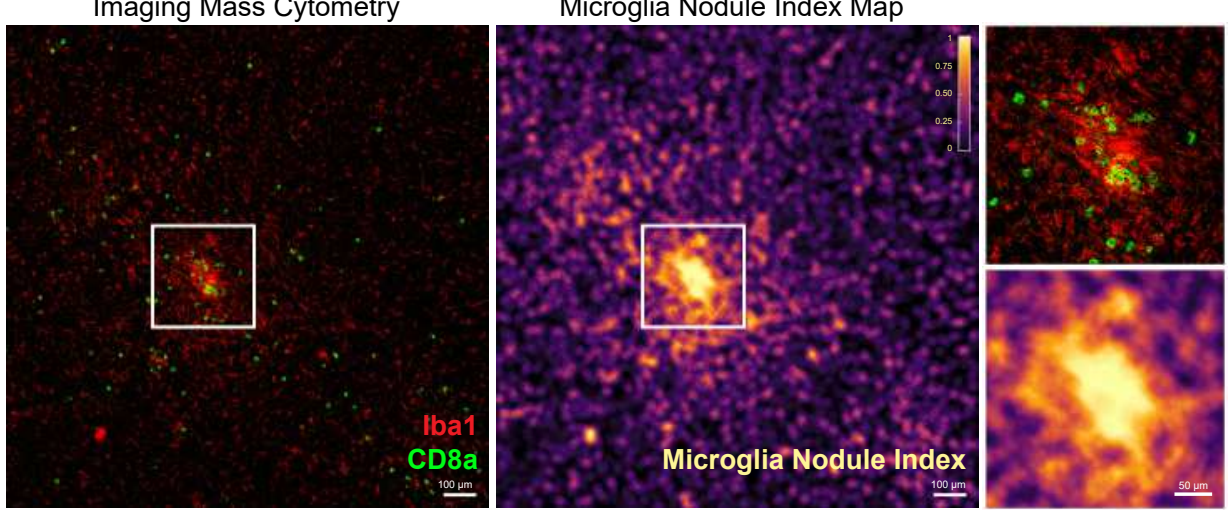

$B$
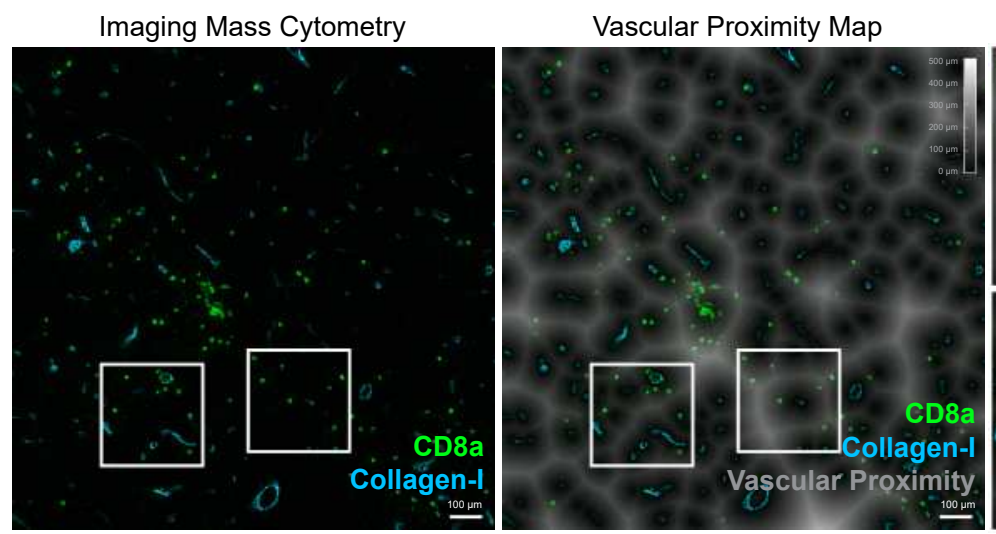

$\mathrm{D}$
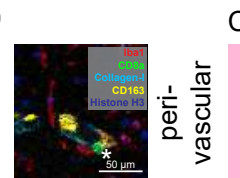

Composition of different CNS compartments

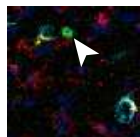

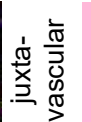
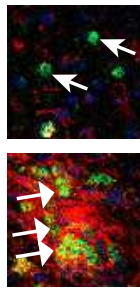

这

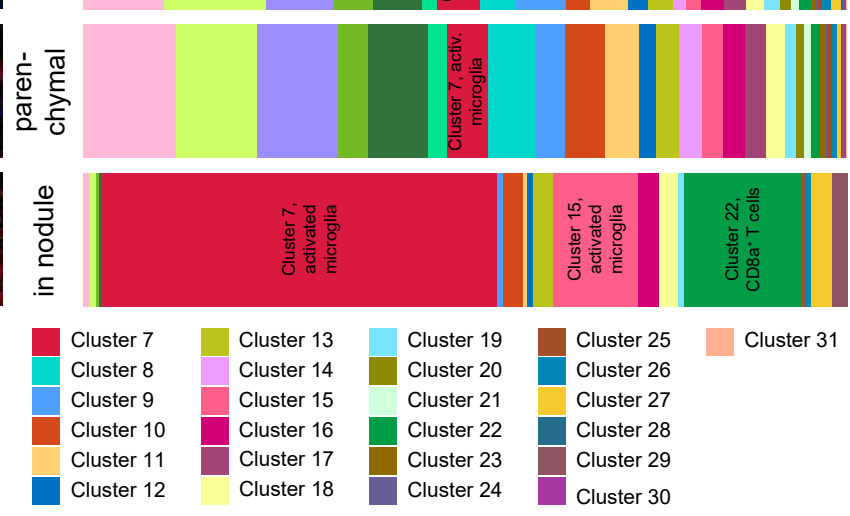

Cluster 1
Cluster 2

Cluster 3

Cluster 4

Cluster 5

Cluster 6
C Microglia alterations: patient scoring

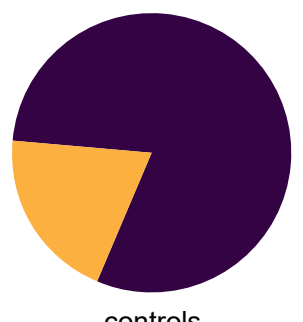

controls

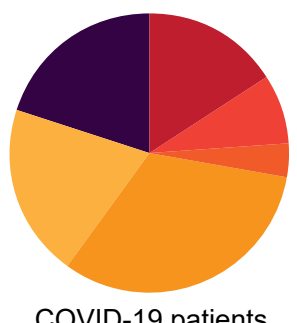

severe microgliosis, microglia nodules

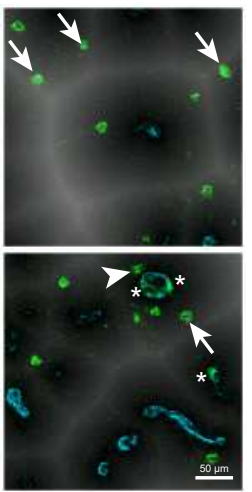

$E$

Cluster distribution across different CNS compartments
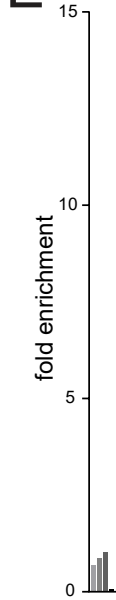

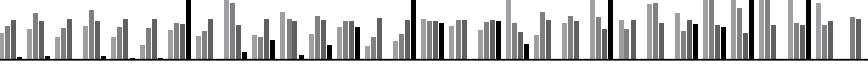

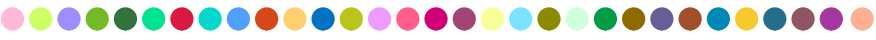

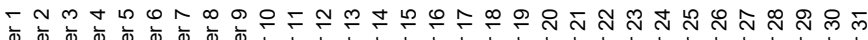

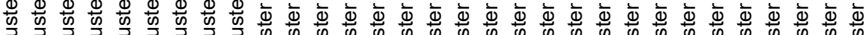

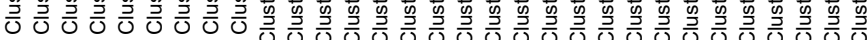
- vascular enrichment parenchymal enrichment juxtavascular enrichment $\mathbf{a}$ nodule enrichment

Fig. 4 
A PD-1 expression
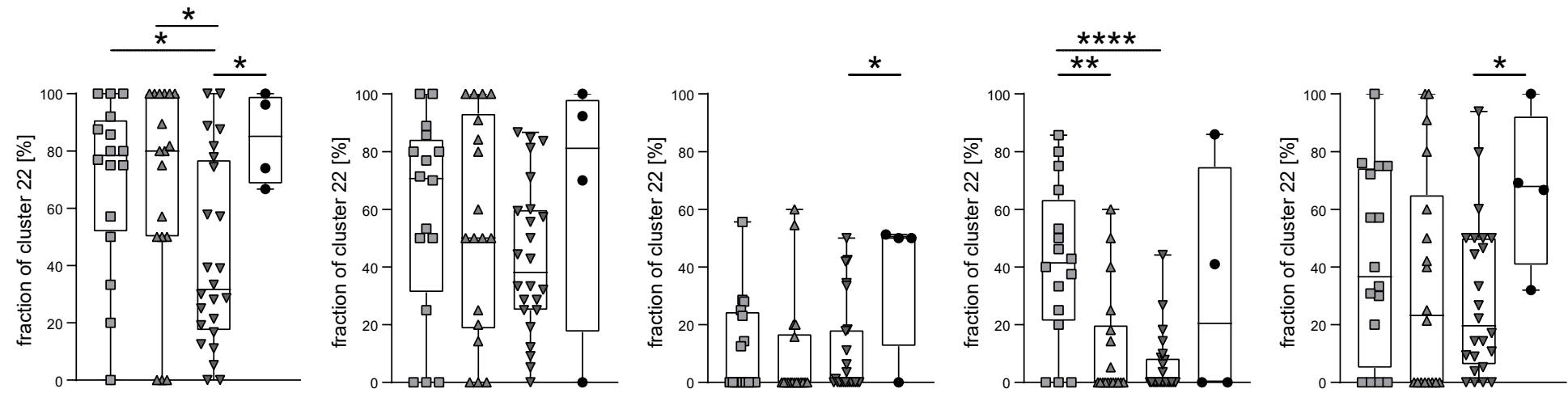

$\square$ perivascular compartment $\quad \Delta$ juxtavascular compartment $\quad \nabla$ parenchymal compartment

nodule compartment

B $\begin{gathered}\text { Spatial HLA-DR } \\ \text { expression heatmap }\end{gathered}$

$\begin{array}{lllllllllll}0 & 1.8 & 3.6 & 5.4 & 7.2 & 9.0 & 10.8 & 12.6 & 14.4 & 16.2 & 18.0\end{array}$

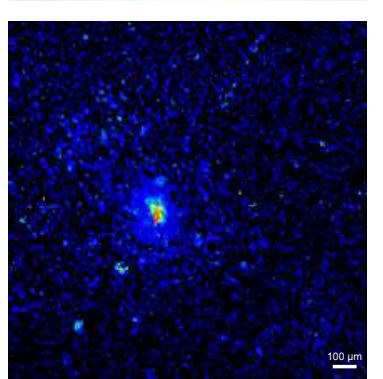

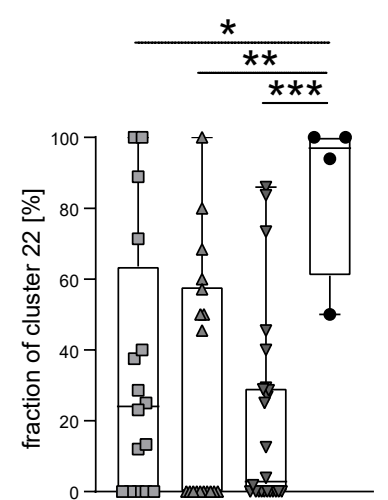
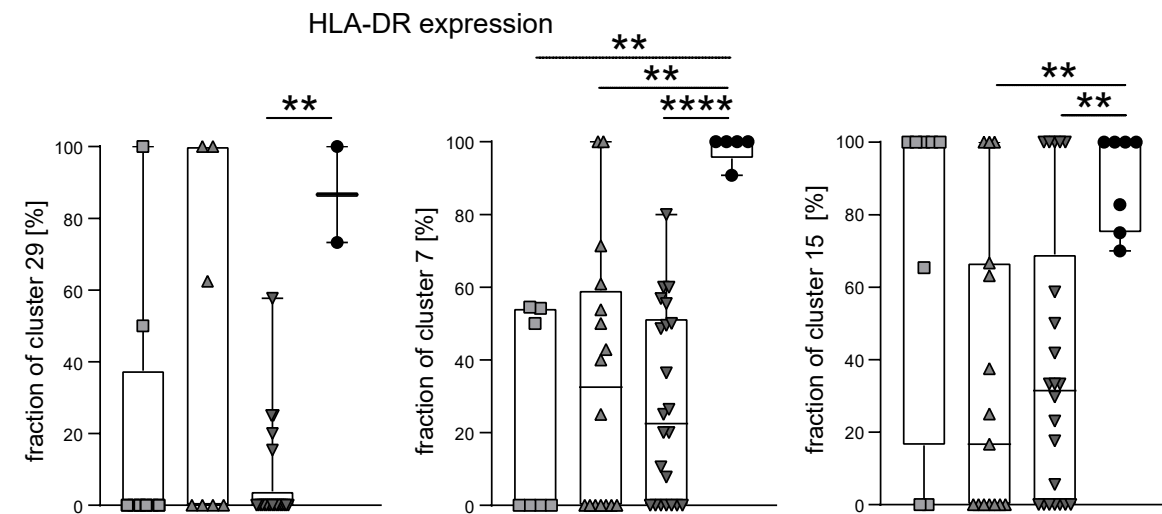

$\square$ perivascular compartment

$\Delta$ juxtavascular compartment

$\nabla$ parenchymal compartment

nodule compartment

C

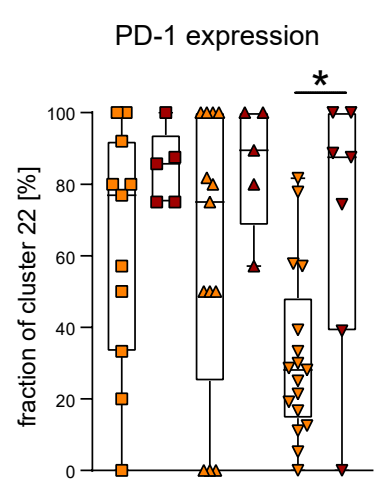

CD39 expression

PD-1 CD39 coexpression

HLA-DR expression

Eomes expression

perivascular compartment in patients without microglia nodules

juxtavascular compartment in patients without microglia nodul

$\stackrel{*}{*} \stackrel{ }{*}$
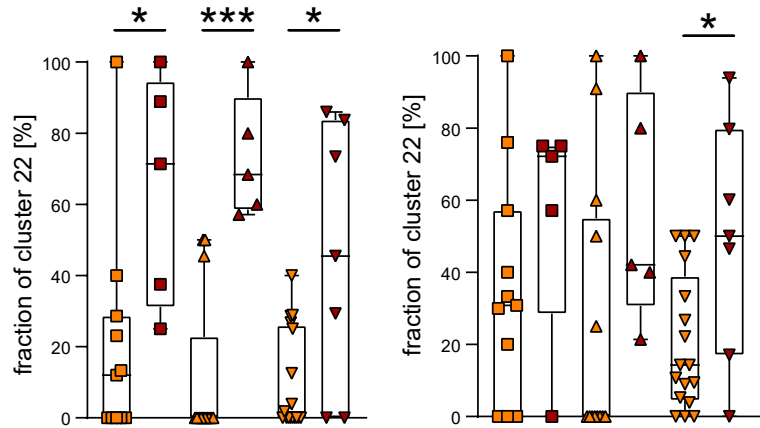

$\square$ perivascular compartment in patients with microglia nodules

A juxtavascular compartment in patients with microglia nodules

$\nabla$ parenchymal compartment in patients without microglia nodules

$\boldsymbol{\nabla}$ parenchymal compartment in patients with microglia nodules

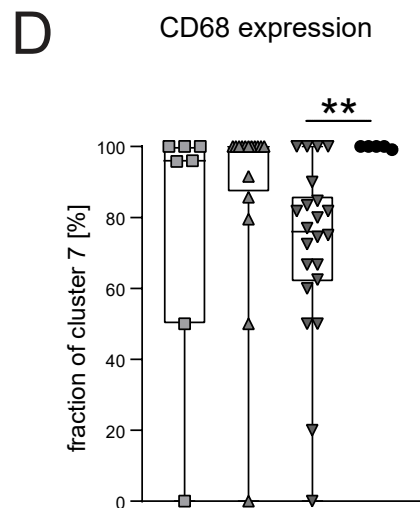

CD33 expression

CD39 expression

Tim3 expression

PD-L1 expression

Fig. 5
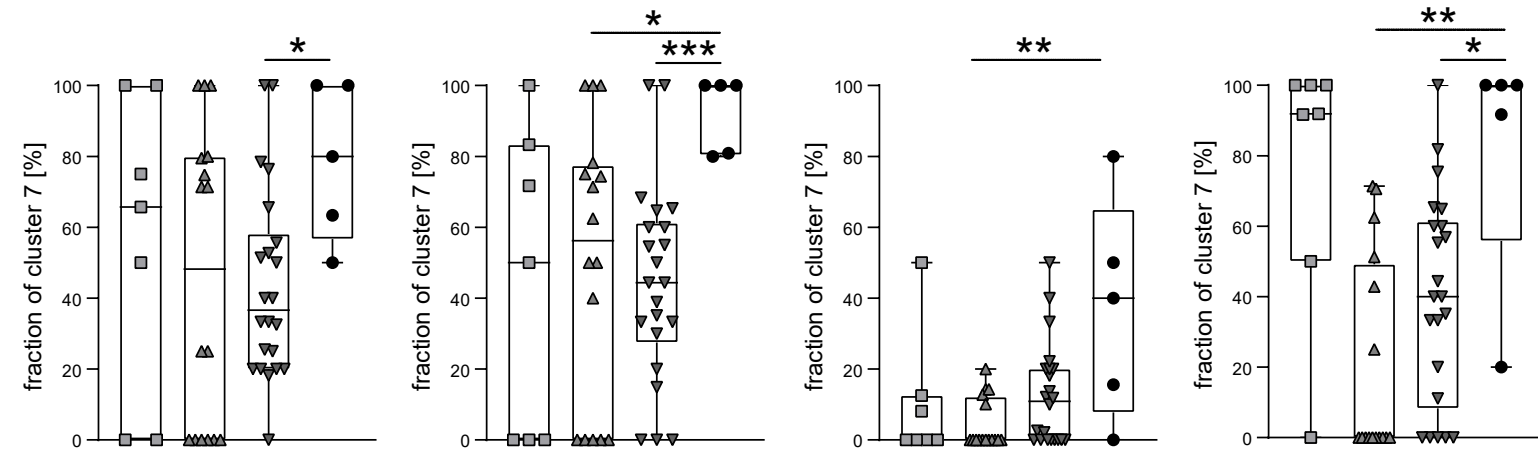

$\square$ perivascular compartment

$\triangle$ juxtavascular compartment

$\nabla$ parenchymal compartment

- nodule compartment 
Cluster 15

Cluster 17

Cluster 17
Cluster 18

Cluster 19
Cluster 20

Cluster 20
Cluster 21

Cluster 22
Cluster 23
Clute

Cluster 24

Cluster 25

Cluster 26

Cluster 27

Cluster 28

Cluster 30

Cluster 31

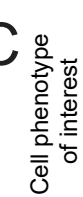

0

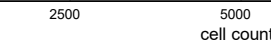

cell count

Cell phenotype in neighborhood

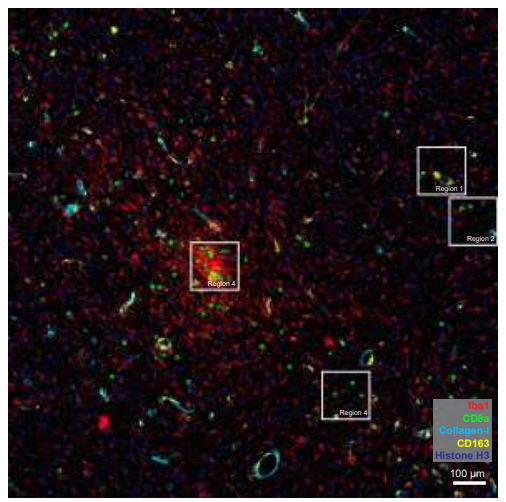

$\mathrm{F}$

CD68 expression

CD33 expression
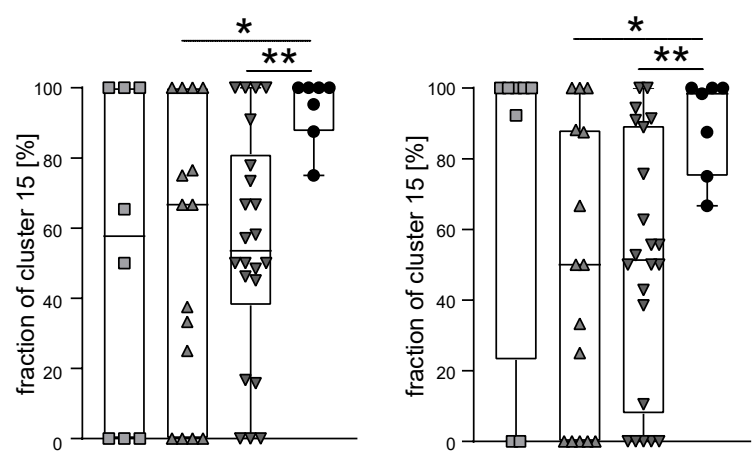

$\square$ perivascular compartment

$\triangle$ juxtavascular compartment

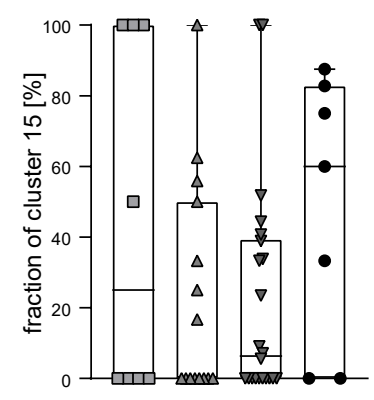

$\nabla$ parenchymal compartment

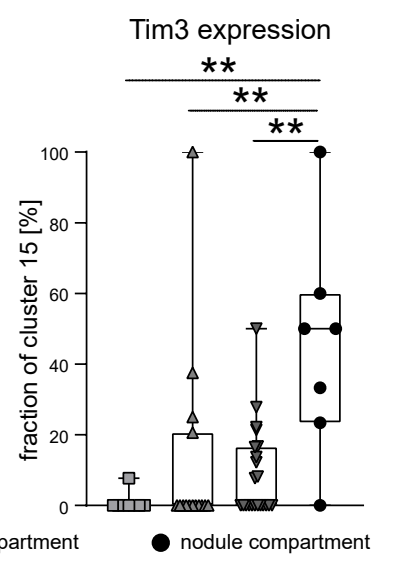

PD-L1 expression

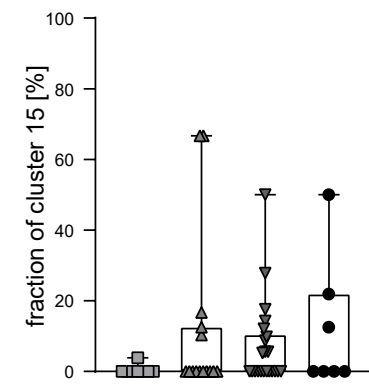

$\mathrm{H}$
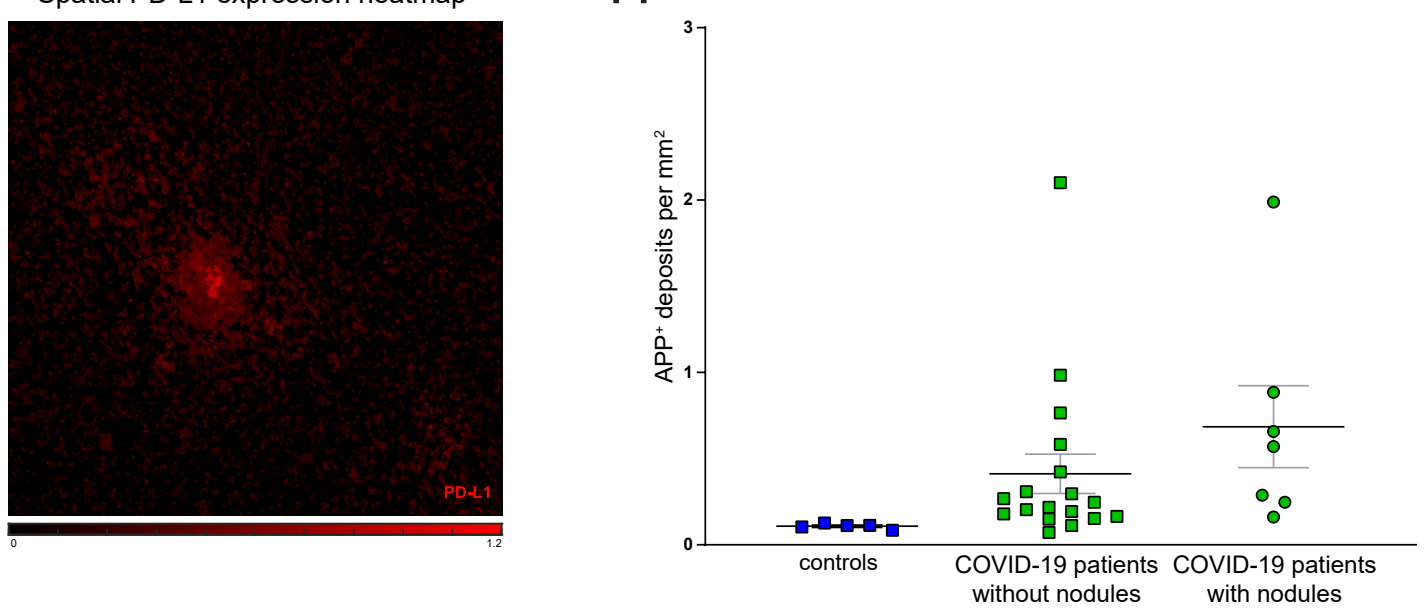

Supplementary Fig. 1 


\begin{tabular}{|c|c|c|c|c|c|c|c|c|c|c|c|}
\hline ID & Group & Sex & Age & POD & PMI & CauseOfDeath & Comorbidities & Brainweight[g] & Brain edema & Brain atrophy & Macroscopic findings \\
\hline 1 & COVID-19 & $\mathrm{m}$ & 75 & $\mathrm{NH}$ & 3 & sudden cardiac death & Parkinson's disease & 1110 & 1 & 0 & none \\
\hline 2 & COVID-19 & $f$ & 87 & $\mathrm{NH}$ & 0 & pneumonia & COPD, dementia, IHD, RI & 1215 & 0 & 1 & none \\
\hline 3 & COVID-19 & $f$ & 85 & hosp. & 0 & pneumonia & atrial fibrillation, $\mathrm{Cl}, \mid \mathrm{IHD}$, myelofibrosis, $\mathrm{RI}$ & 1240 & 0 & 1 & Fresh infarction in territory of ACP \\
\hline 4 & COVID-19 & $f$ & 86 & $\mathrm{NH}$ & 0 & pneumonia & COPD, dementia, IHD & 1250 & 0 & 1 & Fresh infarction in territory of ACP \\
\hline 5 & COVID-19 & $\mathrm{m}$ & 90 & $\mathrm{NH}$ & 2 & pneumonia & atrial fibrillation, dementia, DM, history of stroke & 1015 & 0 & 2 & Old infarctions in territory of ACP \\
\hline 6 & COVID-19 & $\mathrm{m}$ & 90 & hosp. & 3 & emphysema with respiratory decompensation & $\mathrm{Cl}, \mathrm{COPD}$ & 1440 & 0 & 1 & none \\
\hline 7 & COVID-19 & $\mathrm{m}$ & 77 & hosp. & 2 & pneumonia & aortic aneurysm, atrial flutter, cardiac hypertrophy, emphysema, R & 1590 & 5 & 0 & none \\
\hline 8 & COVID-19 & $\mathrm{m}$ & 76 & $\mathrm{ICU}$ & 3 & pe, respiratory tract infection & $\mathrm{Cl}, \mathrm{COPD}$ & 1460 & 1 & 0 & none \\
\hline 9 & COVID-19 & $\mathrm{m}$ & 70 & hosp. & 1 & pneumonia (aspiration) & $\mathrm{Cl}, \mathrm{COPD}, \mathrm{IHD}$, Parkinsons's disease & 1430 & 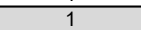 & 0 & none \\
\hline 10 & COVID-19 & $\mathrm{m}$ & 93 & hosp. & 3 & pneumonia & DM, hypertension & 1400 & 1 & 0 & none \\
\hline 11 & COVID-19 & $\mathrm{m}$ & 66 & ER & 2 & pneumonia & $\mathrm{DM}, \mathrm{IHD}$ & 1450 & 1 & 0 & none \\
\hline 12 & COVID-19 & $f$ & 54 & hosp. & 1 & pneumonia & trisomy 21, epilepsy & 950 & 0 & 3 & Grey matter heterotopia \\
\hline 13 & COVID-19 & $\mathrm{f}$ & 86 & $\mathrm{NH}$ & 2 & sepsis, pneumonia & emphysema, epilepsy, hypoxic brain damage, IHD, RI & 1210 & 0 & 1 & none \\
\hline 14 & COVID-19 & f & 85 & home & 1 & pneumonia & $\mathrm{Cl}, \mathrm{COPD}$ & 1180 & 0 & 1 & none \\
\hline 15 & COVID-19 & $\mathrm{f}$ & 70 & ICU & 3 & pneumonia & $\mathrm{Cl}$ & 1150 & 0 & 1 & none \\
\hline 16 & COVID-19 & f & 75 & ICU & 4 & pneumonia & cardiac arrhythmia, IHD & 1210 & 0 & 1 & none \\
\hline 17 & COVID-19 & $\mathrm{m}$ & 93 & hosp. & 2 & pneumonia & atrial fibrillation, $\mathrm{Cl}, \mathrm{IHD}$, obstructive sleep apnoea syndrome & 1000 & 0 & 2 & Old cerebellar infarction \\
\hline 18 & COVID-19 & $\mathrm{f}$ & 82 & hosp. & 4 & purulent bronchitis & COPD, history of pulmonary embolism, RI & 1080 & 0 & 2 & none \\
\hline 19 & COVID-19 & $\mathrm{m}$ & 63 & ICU & 1 & pe, pneumonia & $\mathrm{Cl}$ & 1435 & 1 & 0 & Fresh infarction in territory of ACA \\
\hline 20 & COVID-19 & $\mathrm{m}$ & 84 & hosp. & 5 & pneumonia, septic encephalopathy & $\mathrm{DM}$, history of stroke, hypertension, IHD, ulcerative colitis & 1350 & 1 & 0 & none \\
\hline 21 & COVID-19 & $\mathrm{m}$ & 71 & ICU & 2 & pe, pneumonia & $\mathrm{Cl}$, DM, lung granuloma & 1665 & 2 & 0 & none \\
\hline 22 & COVID-19 & $\mathrm{m}$ & 59 & hosp. & 12 & pneumonia & cardiomyopathy & 1575 & 4 & 0 & none \\
\hline 23 & COVID-19 & $\mathrm{m}$ & 85 & ICU & 2 & pneumonia & COPD, history of aortic valve replacement, hypertension, IHD & 1400 & 1 & 0 & none \\
\hline 24 & COVID-19 & $f$ & 75 & home & 2 & pulmonary artery embolism & hypertension, IHD & 1095 & 0 & 2 & none \\
\hline 25 & COVID-19 & f & 76 & hosp. & 2 & pneumonia & breast cancer, hypertension & 1180 & 0 & 1 & none \\
\hline 26 & control & f & 82 & hosp. & 3 & aspiration & hypertension & 1380 & 0 & 0 & none \\
\hline 27 & control & $\mathrm{m}$ & 81 & hosp. & 2 & respiratory failure / invasive lung carcinoma & lung squamous cell carcinoma, DVT, pulmonary embolisms, $\mathrm{Cl}$ & 1450 & 0 & 0 & none \\
\hline$\frac{21}{28}$ & control & $f$ & 88 & hosp. & $\frac{2}{1}$ & cardiac failure with cardiomegaly & $\begin{array}{c}\text { tension pneumothorax, aortic valve replacement } \\
\text { to }\end{array}$ & 1260 & 0 & 0 & none \\
\hline 29 & control & $\mathrm{m}$ & 87 & hosp. & 3 & hemorrhagic shock / Gl bleeding & atrial fibrillation, anticoagulation & 1110 & 0 & 0 & none \\
\hline 30 & control & f & 78 & hosp. & 2 & ambilateral fulminant pulmonary embolism & hypertension & 1300 & 0 & 0 & none \\
\hline
\end{tabular}

$\mathrm{m}$ : male, f: female

n/a: not available
POD (place of death): ER: Emergency room, hosp.: Hospital ward, ICU: Intensive care unit, NH: Nursing home

cause of death: pe: pulmonary artery embolisms, scd: sudden cardiac death

PMI: postmortem interval (days)

co-morbidities: Cl: cardiac insufficiency, COPD: chronic obstructive pulmonary disease, DM: diabetes mellitus, IHD: ischemic heart disease, RI: renal insufficiency, TX: Transplantation

Brain edema, brain atrophy, arteriosclerosis: 0: none, 1: basal, 2: moderate, 3: severe

Macroscopic findings: ACP: Arteria cerebri posterior, ACA: Arteria cerebri anterior, ACM: Arteria cerebri media 


\begin{tabular}{|c|c|c|c|c|c|}
\hline Metal & Target & Clone & Vendor & Catalog \# & Dilution \\
\hline $89 Y$ & CD45 & D9M8I & CST & 13917BF & $1: 50$ \\
\hline $115 \mathrm{In}$ & HLA-DR & TAL-1B5 & abcam & ab176408 & $1: 400$ \\
\hline $141 \mathrm{Pr}$ & Iba1 & EPR16588 & abcam & ab178846 & $1: 200$ \\
\hline $142 \mathrm{Nd}$ & CD56 & EPR2566 & abcam & $a b 214436$ & $1: 100$ \\
\hline $143 \mathrm{Nd}$ & GFAP & EPR1034Y & abcam & ab218309 & $1: 100$ \\
\hline $144 \mathrm{Nd}$ & CD69 & EPR21814 & abcam & $a b 234512$ & $1: 100$ \\
\hline $146 \mathrm{Nd}$ & CD39 & EPR20627 & abcam & $a b 236038$ & $1: 50$ \\
\hline $147 \mathrm{Sm}$ & CD163 & EDhu-1 & fluidigm & 3147021D & $1: 200$ \\
\hline $148 \mathrm{Nd}$ & NeuN & 1B7 & BioLegend & 834501 & $1: 400$ \\
\hline $149 \mathrm{Sm}$ & CD103 & EPR22590-27 & abcam & $a b 2454201$ & $1: 50$ \\
\hline $150 \mathrm{Nd}$ & PD-L1 & E1L3N & CST & $13684 S$ & $1: 50$ \\
\hline 151Eu & ACE-2 & $\mathrm{AC} 18 \mathrm{~F}$ & Novus & NBP2-80035 & $1: 50$ \\
\hline $152 \mathrm{Sm}$ & TCF-1 & C63D9 & CST & 2203BF & $1: 100$ \\
\hline 153Eu & TOX & NAN448B & abcam & $a b 237009$ & $1: 100$ \\
\hline $154 \mathrm{Sm}$ & Tim-3 & D5D5R & fluidigm & 3154024D & $1: 800$ \\
\hline $155 \mathrm{Gd}$ & FoxP3 & 236A/E7 & Thermo fisher & $14-4777-82$ & $1: 100$ \\
\hline $156 \mathrm{Gd}$ & CD4 & EPR6855 & fluidigm & 3156033D & $1: 400$ \\
\hline $157 \mathrm{Gd}$ & TIGIT & $4 \mathrm{~A} 12$ & Enzo & ENZ-ABS698-0100 & $1: 100$ \\
\hline $158 \mathrm{Gd}$ & IRF8 & 7G11A45 & BioLegend & 656501 & $1: 100$ \\
\hline 159Tb & CD68 & KP1 & fluidigm & 3159035D & $1: 2000$ \\
\hline $160 \mathrm{Gd}$ & T-bet & 4B10 & fluidigm & $3160010 B$ & $1: 100$ \\
\hline 161Dy & CD20 & $\mathrm{H} 1$ & fluidigm & 3161029D & $1: 800$ \\
\hline 162Dy & CD8a & C8/144B & fluidigm & 3162034D & $1: 800$ \\
\hline 163Dy & Eomes & WD1928 & CST & $14-4877-82$ & $1: 100$ \\
\hline 164Dy & Arginase-1 & D4E3M & fluidigm & 316027D & $1: 100$ \\
\hline $165 \mathrm{Ho}$ & PD-1 & D4W2J & CST & 86163BF & $1: 100$ \\
\hline $166 \mathrm{Er}$ & CD204 & J5HTR3 & invitrogen & $14-9054-82$ & $1: 400$ \\
\hline $167 \mathrm{Er}$ & GranzymeB & EPR20129-217 & fluidigm & 3167021D & $1: 1600$ \\
\hline $168 \mathrm{Er}$ & Ki-67 & B56 & fluidigm & $3168022 \mathrm{D}$ & $1: 100$ \\
\hline $169 \mathrm{Tm}$ & Collagen I & Poly & fluidigm & 3169023D & $1: 800$ \\
\hline $170 \mathrm{Er}$ & CD3 & Poly & fluidigm & 3170019D & $1: 200$ \\
\hline $171 \mathrm{Yb}$ & Histone $\mathrm{H} 3$ & $\mathrm{D} 1 \mathrm{H} 2$ & fluidigm & 3171022D & 1:3000 \\
\hline $172 \mathrm{Yb}$ & CD38 & EPR4106 & abcam & $a b 176886$ & $1: 100$ \\
\hline $173 \mathrm{Yb}$ & CD45RO & UCHL1 & fluidigm & 3173016D & $1: 800$ \\
\hline $174 \mathrm{Yb}$ & CD33 & SP266 & abcam & $a b 238784$ & $1: 100$ \\
\hline 175Lu & CD34 & EP373Y & abcam & ab198395 & $1: 400$ \\
\hline $176 \mathrm{Lu}$ & Myelin-CNPase & SMI 91 & BioLegend & 836404 & $1: 200$ \\
\hline
\end{tabular}




\section{Figures}

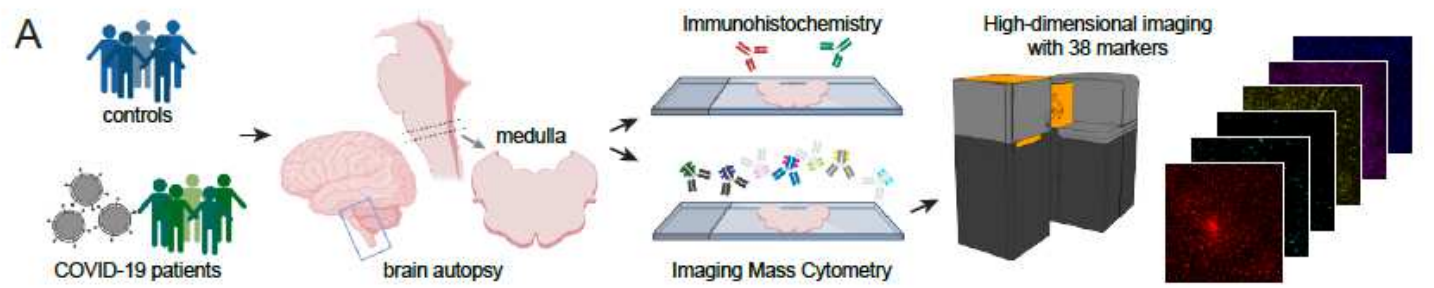

$\mathrm{B}$
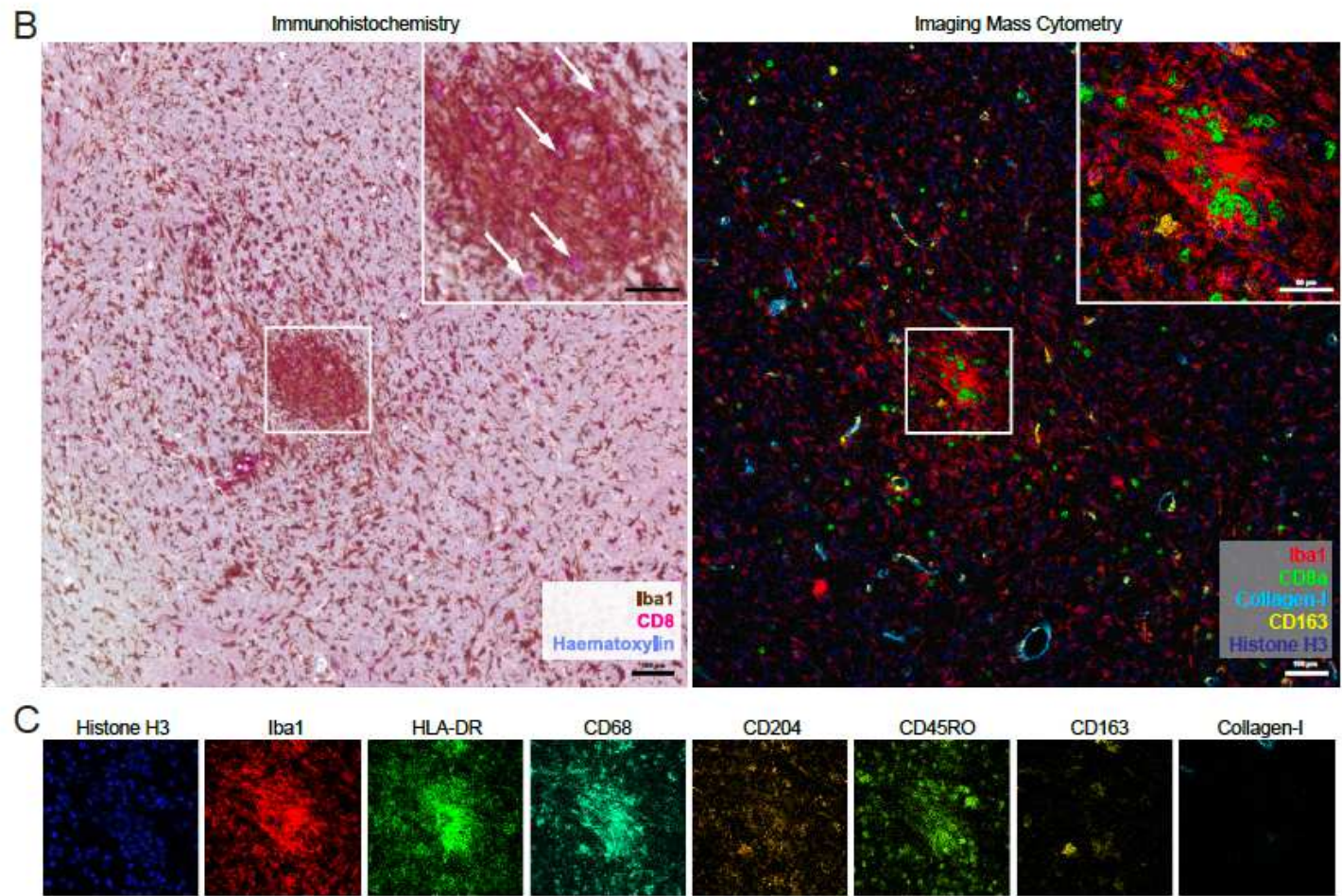

Iba1
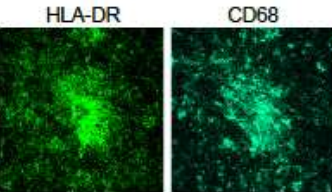

$\mathrm{CD} 204$

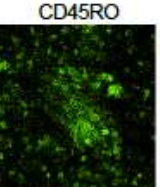

$\mathrm{CD} 163$

Collagen-1
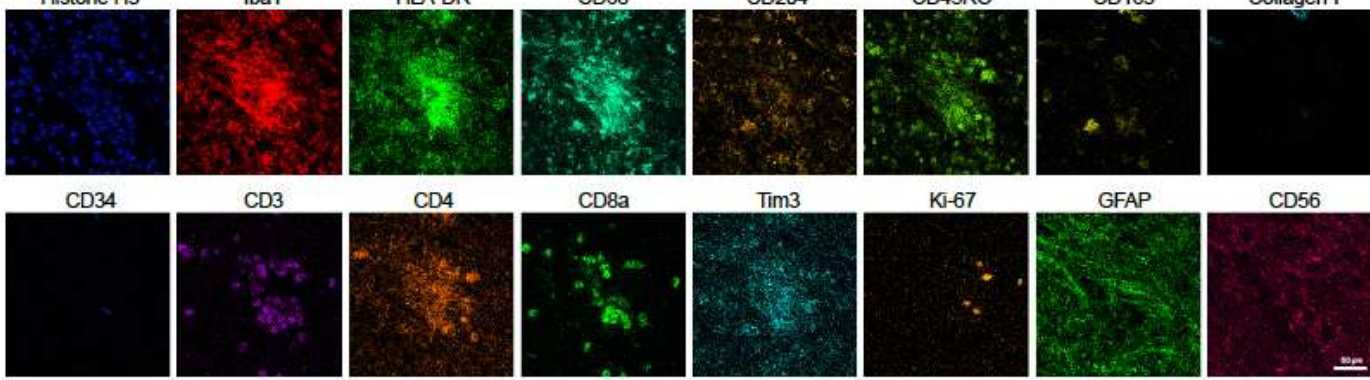

$\operatorname{CD} 56$
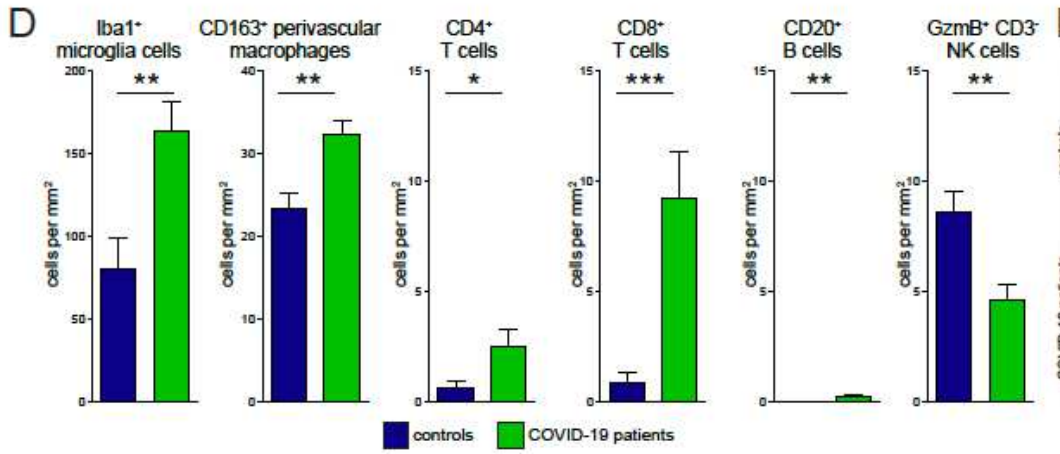

\section{E}

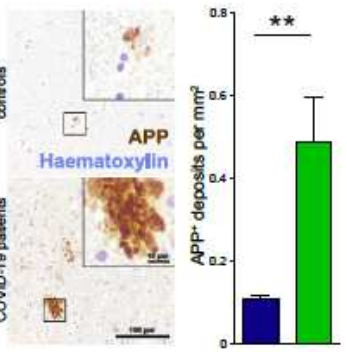

controls $\square$ coviD-19 patients

\section{Figure 1}

Highly multiplexed imaging mass cytometry analysis of COVID-19 brains. A: Graphical illustration of the experimental workflow. Medulla oblongata tissue slices from autopsies of COVID-19 patients $(n=25)$ and controls $(n=5)$ underwent parallel conventional immunohistochemistry and high-dimensional imaging 
mass cytometry (IMC) with 38 markers. B: Exemplary immunohistochemistry (left) and corresponding IMC visualization (right) of the upper medulla tissue of a COVID-19 patient. The area of interest is magnified in the right upper corner. Scale bars indicate $100 \mu \mathrm{m}$ and $50 \mu \mathrm{m}$, respectively. Left: Doublechromogenic immunohistochemical reaction for lba1 (brown) and CD8 (pink), counterstained with haematoxylin (blue). Arrows indicate CD8a+ T cells. Right: Image overlay visualization of Iba1 (red), CD8 (green), collagen-I (light blue), CD163 (yellow) and histone H3 (blue) IMC data out of the 38-marker dataset. C: Visualization of the indicated marker expression data from channels shown exemplarily for one area of interest as indicated in B). The scale bar represents $50 \mu \mathrm{m}$. D: Manual counts of microglial cells, perivascular macrophages, CD4, CD8 T cells, B cells and NK cells was performed on the IMC dataset and compared between controls (blue) and COVID-19 patients (green). Data are shown as bar graphs \pm SEM. E: Robust axonal damage in COVID-19 brains: immunohistochemical reaction for APP (brown) in the upper medulla of control and COVID-19 patients. Counterstaining with hematoxylin (blue). Scale bars indicate $100 \mu \mathrm{m}$ and $10 \mu \mathrm{m}$ in the inserts, respectively. The quantification of APP-positive deposits in the whole section is shown next to the histological images. Data are shown as bar graphs \pm SEM. Significant differences were determined by unpaired $t$ tests with Welch's correction $(D, E)$. In $D$ all controls were negative for manual B cell counts, therefore a one sample $t$ test was used here. 
A

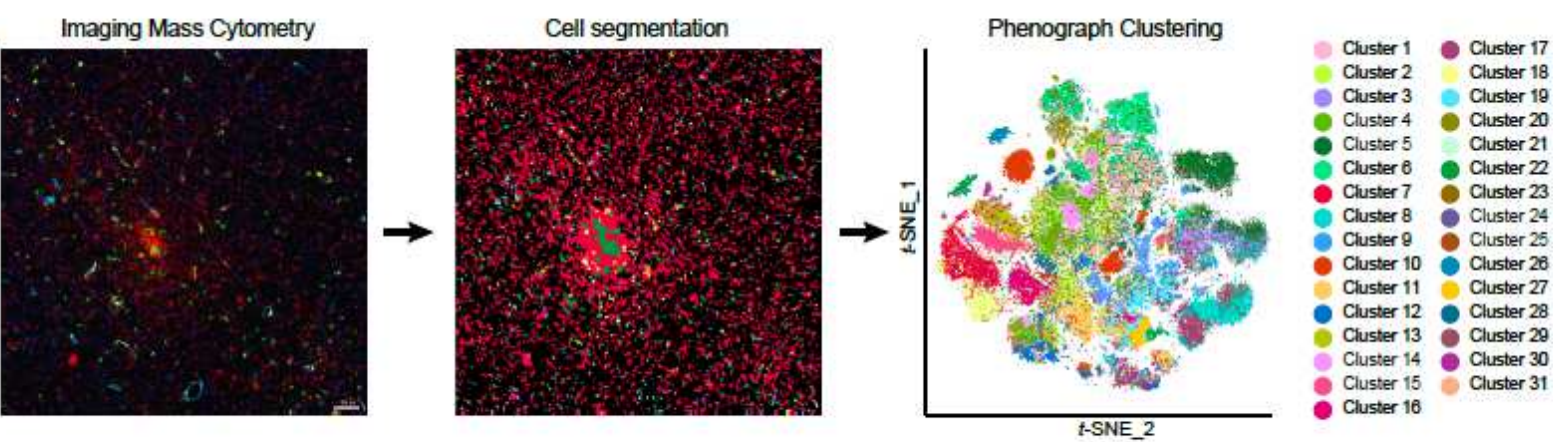

B

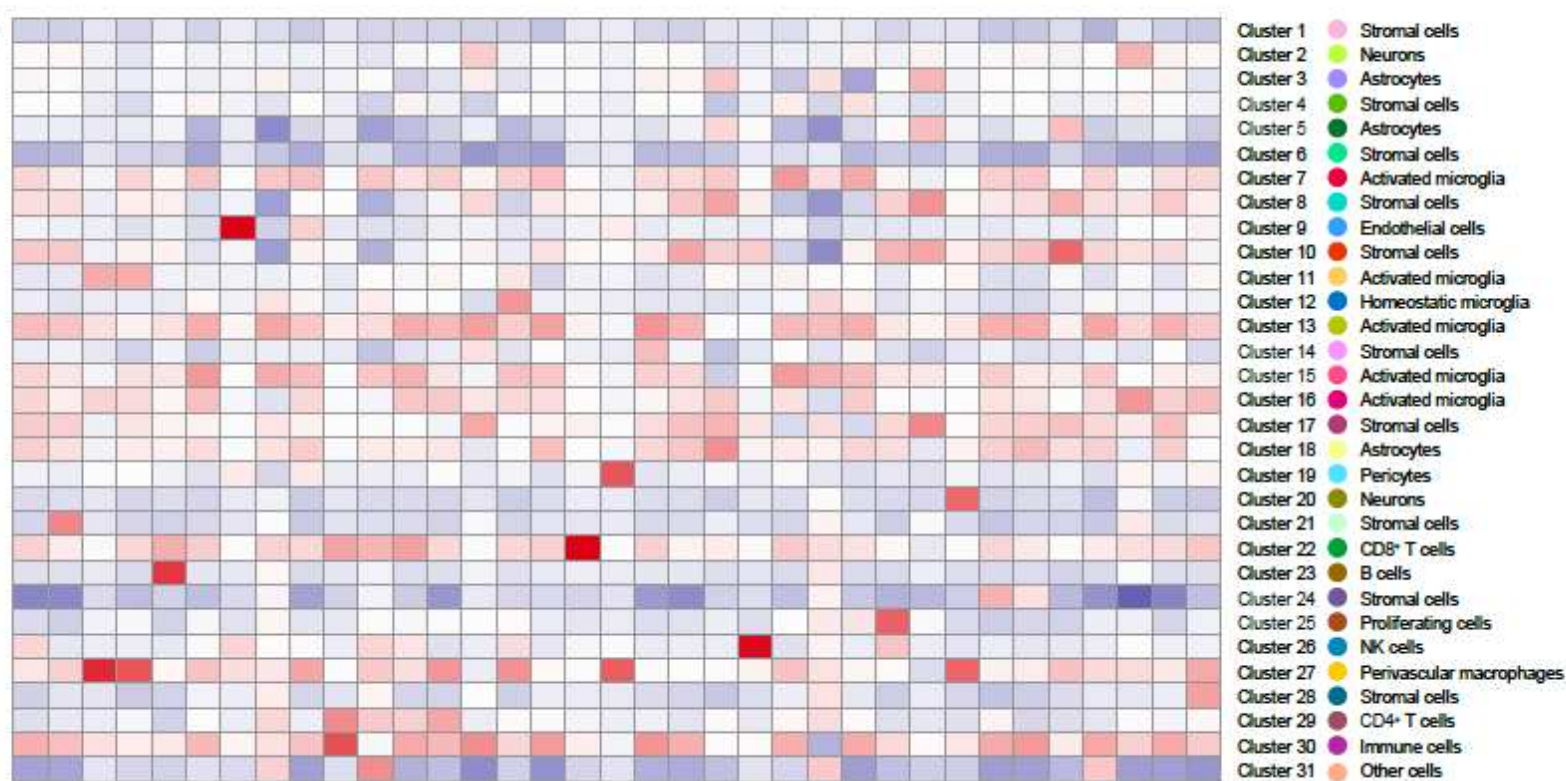

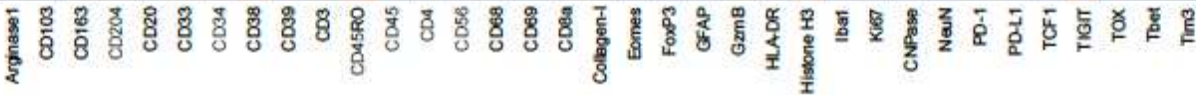

C

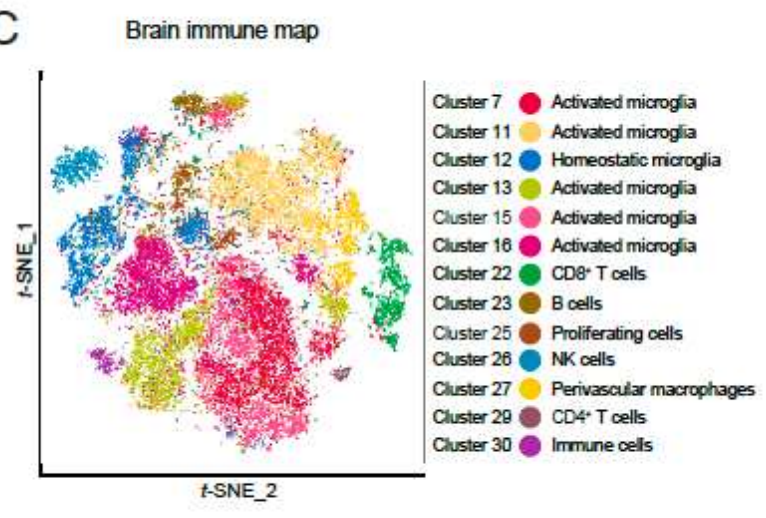

D Cluster composition

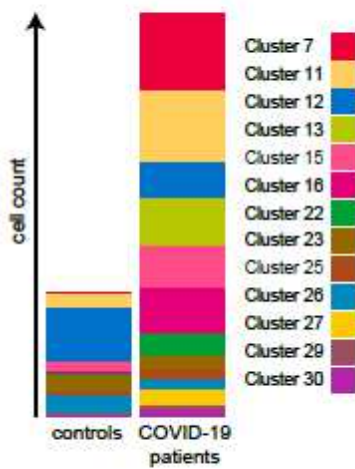

E Immune cell clustering

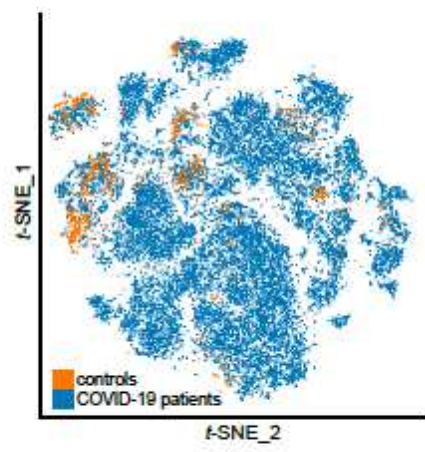

Figure 2

Molecular and cellular census of neuroinflammation during SARS-CoV-2 infection. A: Segmentation of the highly multiplexed IMC images into cellular masks representing single cells was performed on all patients and controls using supervised machine learning. Single-cell data was extracted and cells were clustered using Phenograph and visualized on a t-SNE map. B: Heatmap displaying mean marker expression (shown as z-score) of the Phenograph clusters. Clusters were annotated using the expression 
and spatial distribution of the cells. C: The neuroimmune landscape of SARS-CoV-2 infected and noninfected patients was visualized by t- SNE based on the immunological clusters determined in B. D: The immune cell cluster composition was assessed between the two groups. Stacked bar charts indicate mean counts per group. $\mathrm{E}$ : The brain immune map from $\mathrm{C}$ is plotted according to cellular origin: cells from COVID-19 patients are depicted in blue; cells from controls are shown in orange.

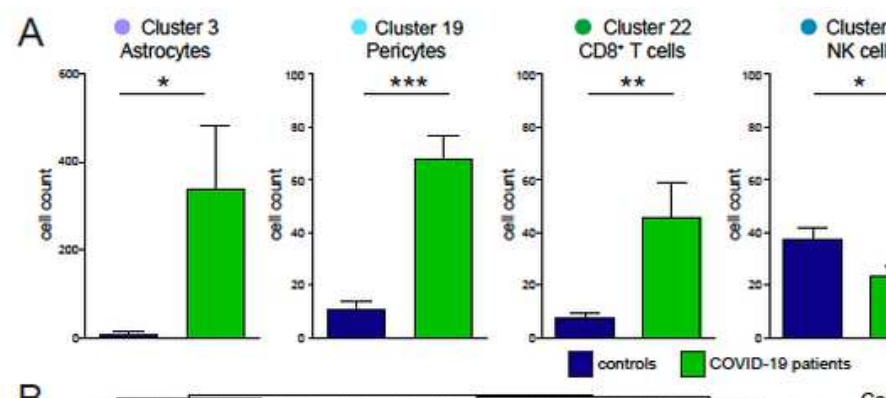

B

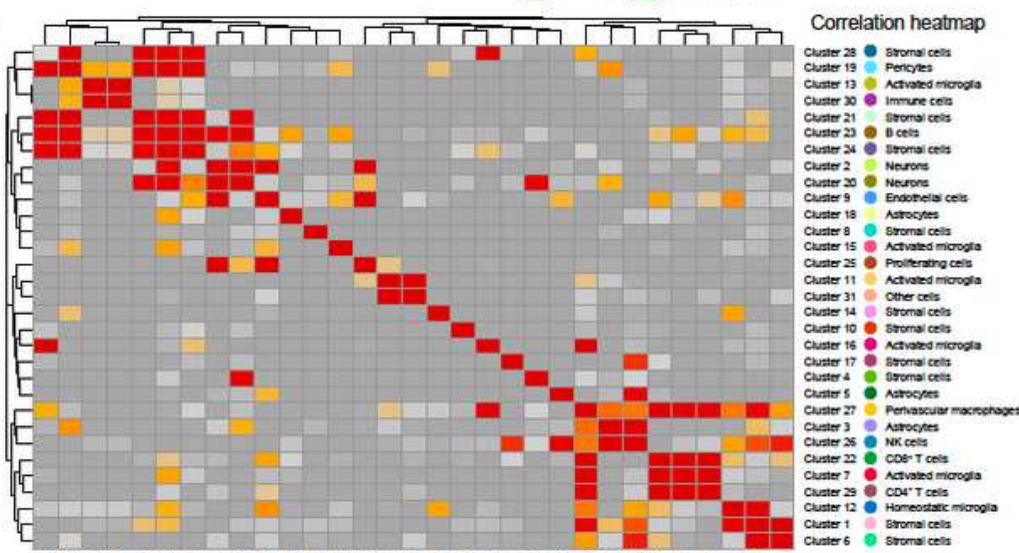

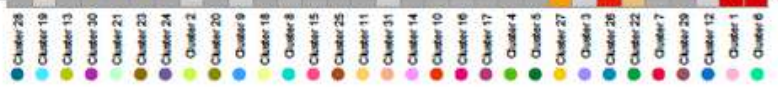

D

Cell phenotype in neighborhood

Neighborhood heatmap
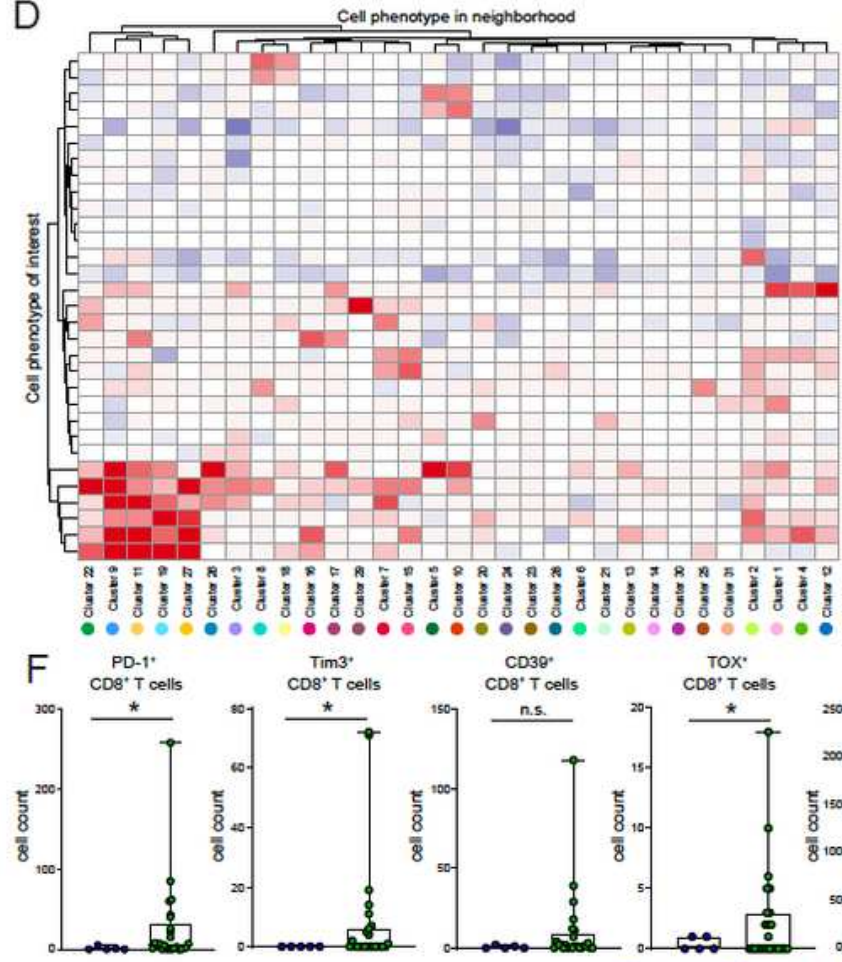

civiter s sromalcets.
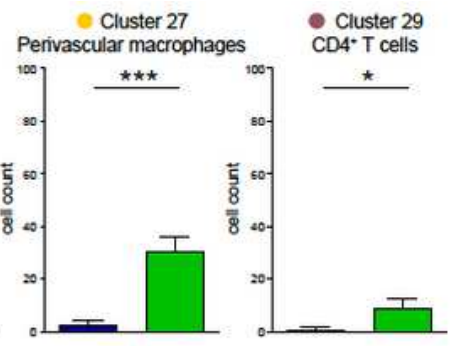

C CD8a T cell distribution

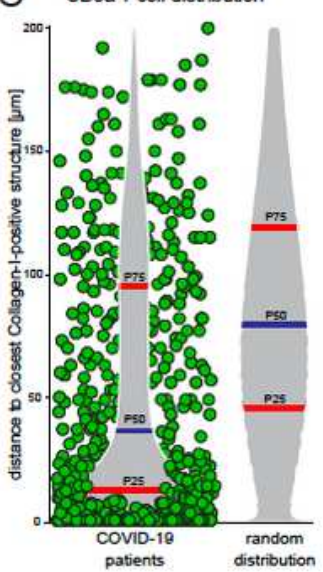

E Interactome cluster 22

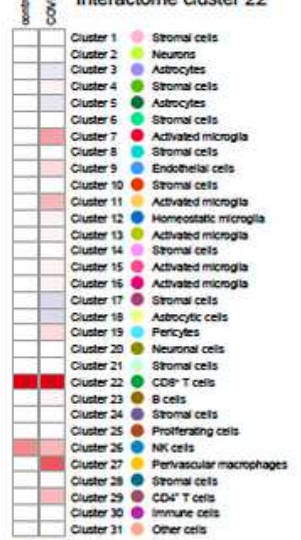

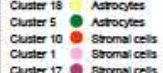

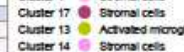

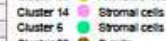

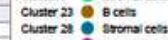

cinter 2 Nesoses

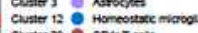

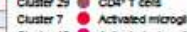

Custer 16 O Actuard mionol

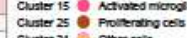

Cunter 200 Nentroral cel

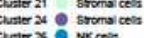

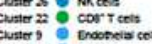

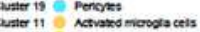

을

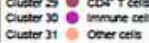

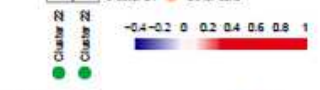

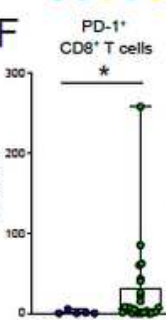

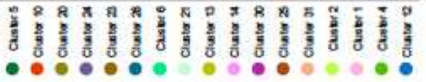

$-0.4-02002040608$

GzmB'
CD8'T cells

$\mathrm{CD}^{\circ} \mathrm{O}^{\circ}$
$\mathrm{CD} 8^{*} \mathrm{~T}$ cells

CDE' $T$ cells

Eomes'
CD8 $T$ cells
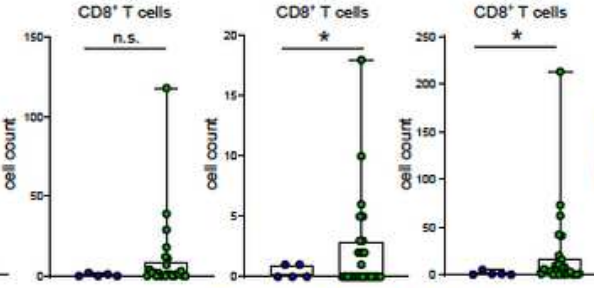

- controls O covid-19 patients

Fig. 3

Figure 3 
Profiling of the CNS immune response during COVID-19 indicates a localized and orchestrated adaptive immune infiltration. A: High-dimensional annotated cells were compared across patients and controls. Typical cell clusters with significantly different abundances are displayed. Means are presented as bar graphs, error bars are indicating \pm SEM. B: Pairwise linear regression analysis between all cluster frequencies in the brains of COVID-19 patients was conducted and p-values of individual cluster pairs were visualized in a heatmap. C: Violin plot indicating the distance of CD8+ cells to the nearest vessel (collagen-I-positive) structure in COVID-19 patients. A random distribution is shown for comparison. The 25th, 50th and 75th percentiles are depicted. The CD8 T cell distribution is further visualized by an overlaid scattered dot plot, showing all CD8+ cells and the corresponding distance to the closest vessel structure in 25 COVID-19 patients. Each dot represents one cell. D: Spatial interactions between cell clusters were assessed in COVID-19 patients by permutation based neighborhood analysis and displayed as hierarchically clustered heatmap of the neighborhood interaction (fraction of significant images) ranging from -1 (avoidance) to +1 (preferential interaction). Rows indicate the neighborhood of a cell phenotype of interest. Columns indicate the enrichment or depletion of a cell in other neighborhoods. $\mathrm{E}$ : Cluster 22 spatial neighborhood interactions were determined in COVID-19 and control patients. Rows indicate the neighborhood of a cell phenotype of interest. F: CD8 T-cell activation in the brains of COVID19 patients. Cluster 22 cells were tested for expression of immune markers involved in T-cell activation, differentiation, exhaustion and function. Absolute cell counts were compared between patient groups. Data are presented as boxplots showing the median, IQR and upper and lower whiskers and dots represent samples. Significant differences were determined by unpaired $t$ tests with Welch's correction (A, F). 
A. Imaging Mass Cytometry

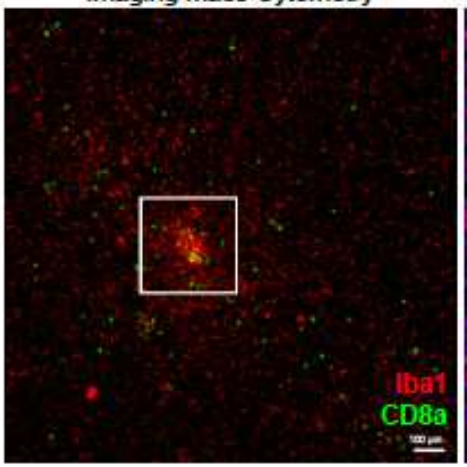

B
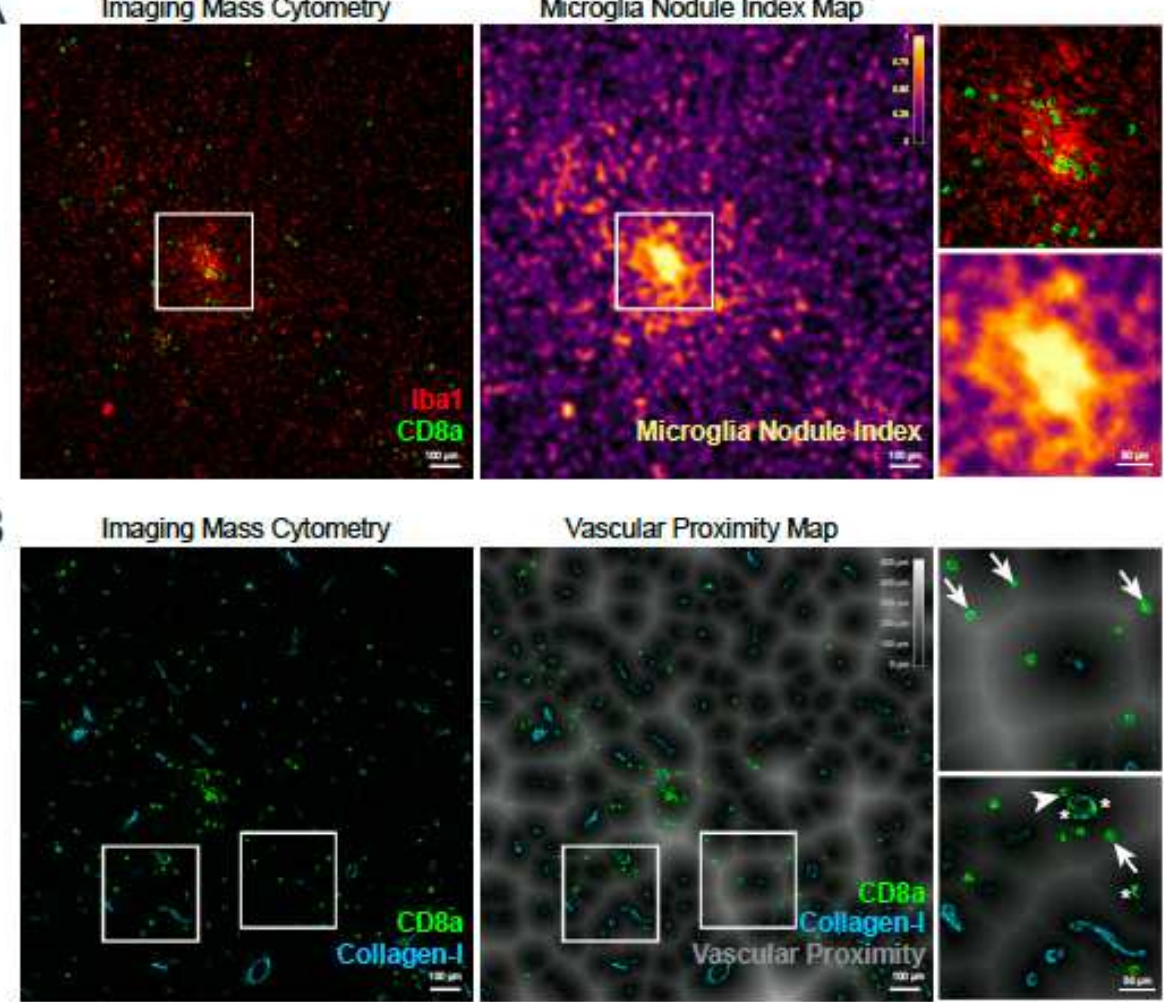

C Microglia alterations: patient scoring

D
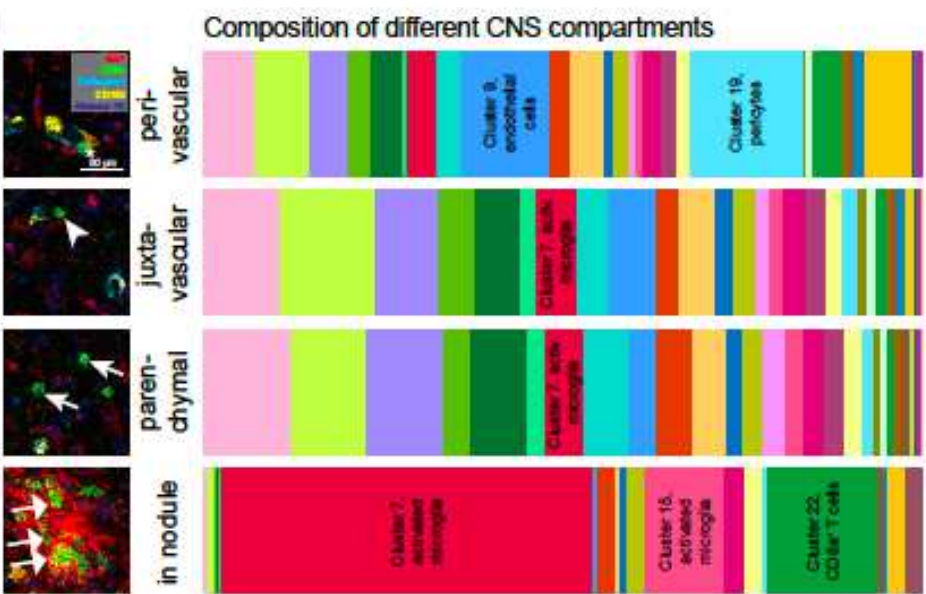

Cluster 1

Custer 2

Custer 3

Custer 4

Custer 5

Custer 6

\section{올}

\section{Cluster 7}

Cluster 7

Cluster 9

Cluster 10

Cluster 11

Cluster 12

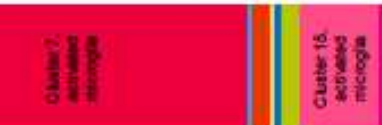

Cluster 13 Cluster 14 Cluster 15 Cluster 16

Cluster 17 Cluster 18

\begin{tabular}{l|l|l|}
\hline Cluster 19 & Cluster 25 & Cluster 31 \\
Cluster 20 & Cluster 26 \\
Cluster 21 & Cluster 27 \\
\hline Cluster 22 & Cluster 28 \\
Cluster 23 & Cluster 29 \\
Cluster 24 & Cluster 30 \\
\hline
\end{tabular}

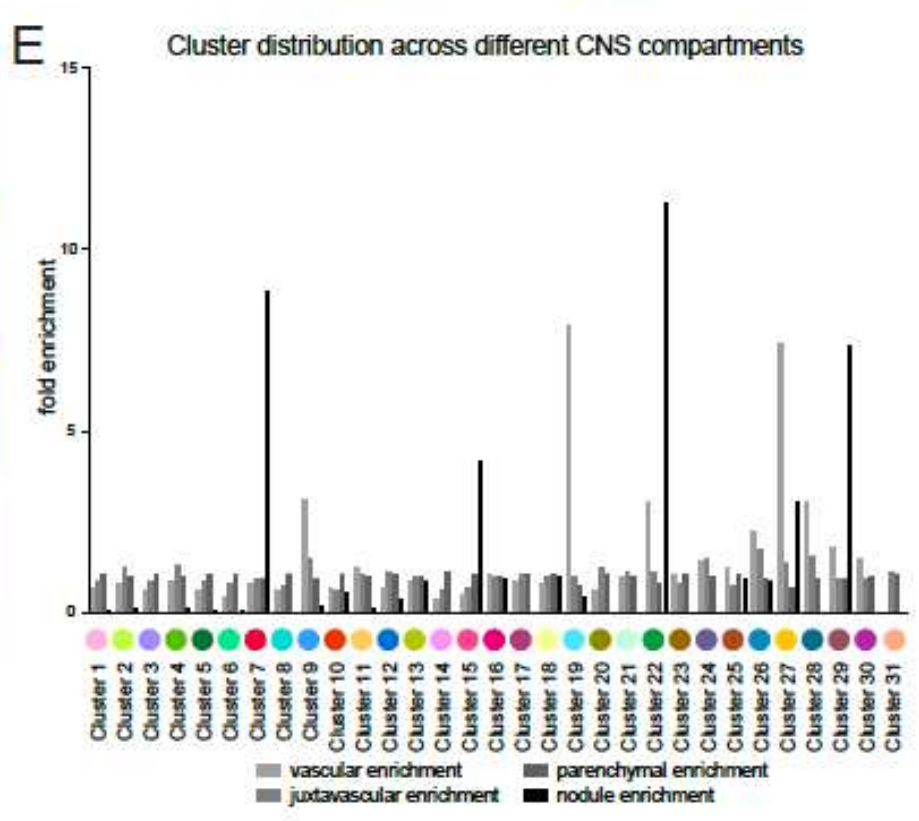

\section{Figure 4}

Spatial compartmentalization of immune cell clusters to distinct anatomical niches in the brain of COVID19 patients. A: Left: Representative IMC image showing Iba1 (red) and CD8a (green) expression. Middle: Microglial distribution was assessed using the accumulation of Iba1 signal across a $15 \mu \mathrm{m}$ and visualized in a microglia nodule index map. Right: magnified inserts of the area of interest indicated in the left and middle panel. Scale bars indicate $100 \mu \mathrm{m}$ and $50 \mu \mathrm{m}$, respectively. B: Vascular distance was estimated as distance to collagen-I. A proximity map displaying the distance of each pixel to the next 
Collagen-l-positive structure was generated and overlaid with collagen-l expression signal. Close proximity is indicated by increasing darkness. Collagen-I expression is indicated in blue. C: Patient sections were assessed for presence of microgliosis or microglial nodules and visualized in a pie chart. There was a significant difference in the prevalence of microglial alterations (microgliosis and/or nodules) between the two groups. D: Cluster composition of anatomical compartments. Images show examples of anatomical niches in one patient sample. Image overlay visualization of Iba1 (red), CD8a (green), collagen I (light blue), CD163 (yellow) and histone H3 (blue). Scale bar indicates $50 \mu \mathrm{m}$. Arrows and asterisks point to CD8 T cells located inside a vessel (perivascular), close to a vessel (juxtavascular), infiltrating into the parenchyma (parenchymal) and inside a microglial nodule (nodule compartment), respectively. Cluster composition of the four compartments was evaluated for all COVID-19 patients and displayed as stacked bar graphs of cluster frequencies per anatomical compartment. E: Enrichment of individual clusters in anatomic compartments was assessed by calculating and visualizing the enrichment index (mean cluster frequency in given compartment / mean frequency in the total COVID- 19 cohort). Unpaired t test with Welch's correction was used in B. 


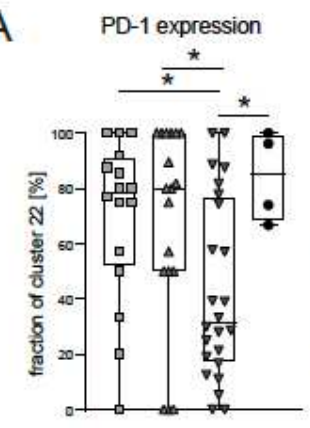

CD39 expression

PD-1 CD39 coexpression

Tim3 expression

Eomes expression
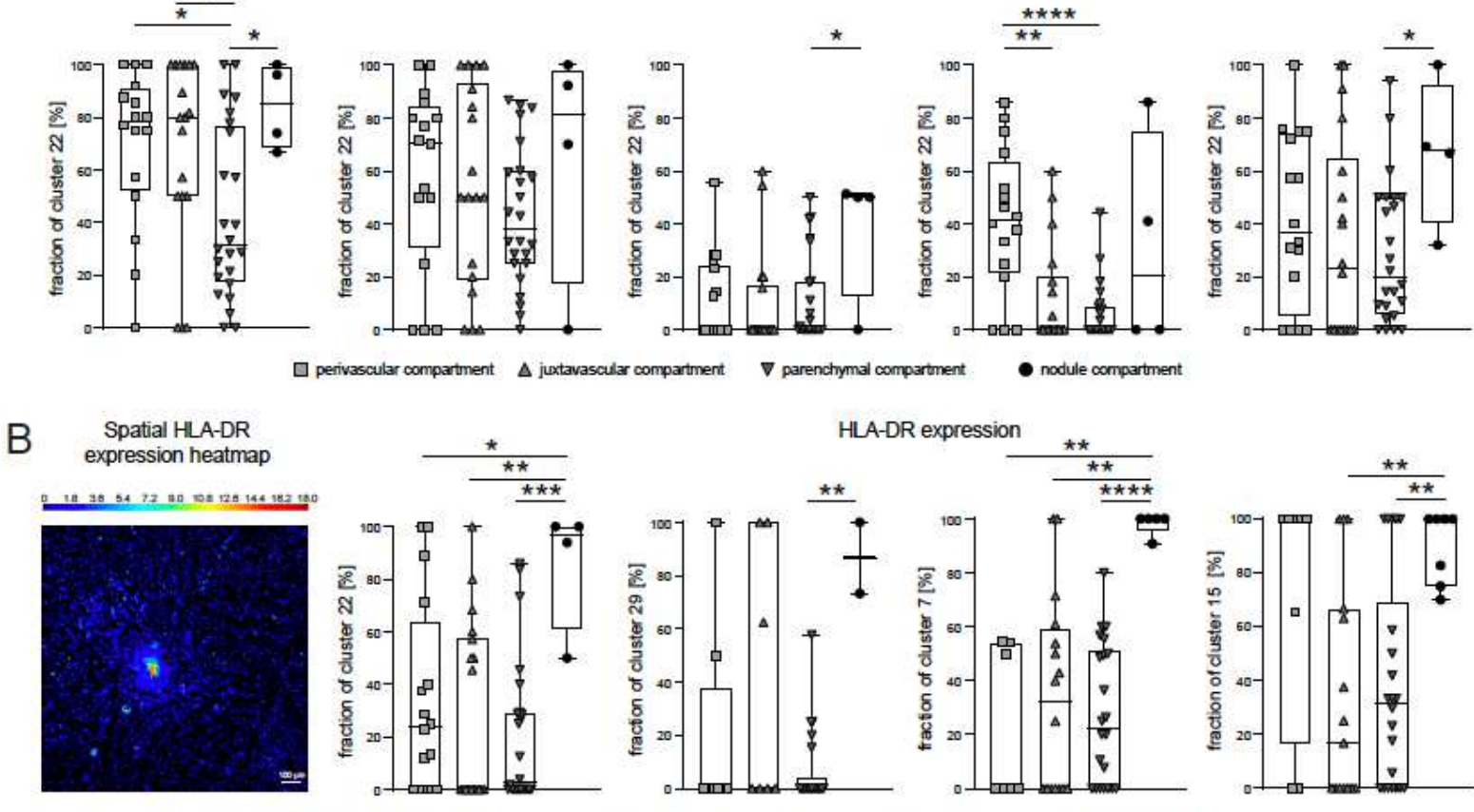

$\square$ perivascular compartment $\Delta$ juxtavascular compartment $\quad \nabla$ parenchymal compartment $\quad$ nodule compartment

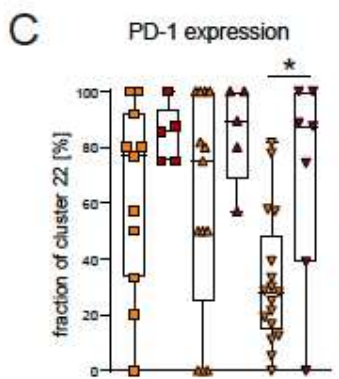

CD39 expression
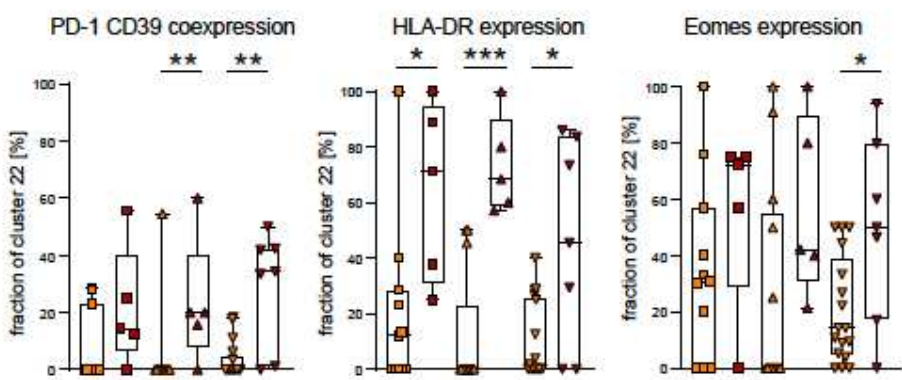

口 pervascular compartment in patients without microgia nodules $\Delta$ juxtavascular compartment in patients without microgla nodules

perivascular compartment in patients with microgia nodules

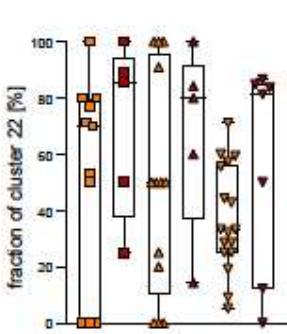

juxtavascular compartment in patients with microglia nodules.

$\nabla$ parenchymal compartment in patients without microglia nodules

V parenchymal compartment in patients with microglia nodules

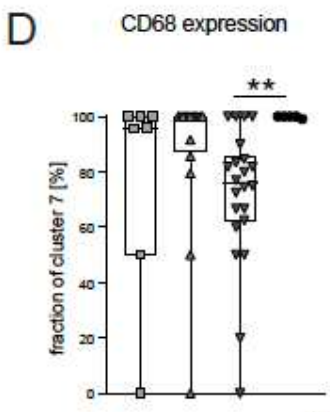

CD33 expression

CD39 expression

Tim3 expression

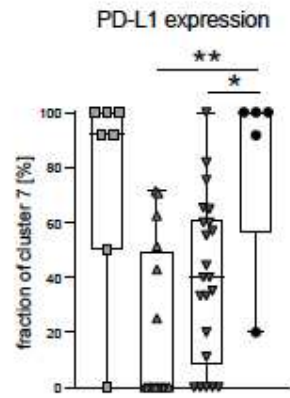

\section{Figure 5}

Assessment of immune cell activation in different anatomical compartments suggests pervasive inflammatory effect of microglial nodules on distant T-cell activation and immune checkpoint expression. A: Analysis of CD8 T cells in different CNS compartments. Frequencies of PD-1+, CD39+, PD-1+ CD39+, Tim3+ and Eomes+ and HLA-DR+ cells of cluster 22 were compared across perivascular, juxtavascular, parenchymal and nodule compartments. Boxplots show the median with IQR and upper and lower 
whiskers. B: HLA-DR expression of CD8 T cells, CD4 T cells and activated microglial cells in different CNS compartments. Left: Spatial heatmap displaying HLA-DR signal intensities in segmented cells on the image. Scale bar indicates $100 \mu \mathrm{m}$. Right: Frequencies of HLA-DR+ cells of cluster c22 (CD8 T cells), c29 (CD4 T cells), c7 (activated microglia) and c15 (activated microglia) compared across perivascular, juxtavascular, parenchymal and nodule compartments. Boxplots show the median with IQR and upper and lower whiskers. C: CD8 T-cell activation in separate anatomical regions. Patients were grouped based on the presence or absence of microglial nodules in the image and the frequencies of PD-1+, CD39+, PD$1+C D 39+$, Eomes+ and HLA-DR+ of cluster c22 were compared between those patients in the perivascular, juxtavascular and parenchymal compartment to reveal a pervasive effect of microglial nodules on the activation of CD8 T cells. Boxplots show the median with IQR and upper and lower whiskers. D: Compartment-dependent expression of activation and immunoregulatory molecules by activated microglial cells. Frequencies of CD68+, CD33+, CD39+, Tim-3+ and PD-L1+ cells of cluster C7 were compared across the four different CNS compartments. Boxplots show the median with IQR and upper and lower whiskers. E: Exemplary image showing PD-1 (blue) and PD-L1 (red) expression on a spatial heatmap of segmented cells. Mann-Whitney-U non-parametric t-tests were used in A-D.

\section{Supplementary Files}

This is a list of supplementary files associated with this preprint. Click to download.

- GraphicalAbstract.png

- SupplementaryData.pdf 